\title{
AN $h p$-LOCAL DISCONTINUOUS GALERKIN METHOD FOR SOME QUASILINEAR ELLIPTIC BOUNDARY VALUE PROBLEMS OF NONMONOTONE TYPE
}

\author{
THIRUPATHI GUDI, NEELA NATARAJ, AND AMIYA K. PANI
}

\begin{abstract}
In this paper, an $h p$-local discontinuous Galerkin method is applied to a class of quasilinear elliptic boundary value problems which are of nonmonotone type. On $h p$-quasiuniform meshes, using the Brouwer fixed point theorem, it is shown that the discrete problem has a solution, and then using Lipschitz continuity of the discrete solution map, uniqueness is also proved. $A$ priori error estimates in broken $H^{1}$ norm and $L^{2}$ norm which are optimal in $h$, suboptimal in $p$ are derived. These results are exactly the same as in the case of linear elliptic boundary value problems. Numerical experiments are provided to illustrate the theoretical results.
\end{abstract}

\section{INTRODUCTION}

In recent years, greater attention has been paid on the application of the discontinuous Galerkin (DG) methods to a wide range of partial differential equations. This is due to their flexibility in locally mesh adaption and their local conservation properties. Since these methods deal with discontinuous finite element spaces, it is easy to allow hanging nodes in the mesh, which is an advantage for the adaptive methods. In literature, there are various DG formulations which have appeared for the elliptic problems; see 3 . The local discontinuous Galerkin (LDG) method is originally initiated for a system of first order hyperbolic problems. The method is carried to elliptic problems for mixed discontinuous Galerkin formulation; see 9 . In [9, the authors discussed stability and order of convergence of the LDG method applied to the Laplace equation. In [7, the LDG method is applied to a quasilinear elliptic problem of the following type:

$$
-\nabla \cdot \mathbf{a}(\cdot, \nabla u)=f \text { in } \Omega
$$

with mixed boundary conditions, where $\mathbf{a}$ is uniformly monotone. Under the assumption that the nonlinear operator induced by $\mathbf{a}$ is monotone, it is shown in [7. that the primal form of the LDG method is monotone. Then, existence of an approximate solution for the LDG method is proved and a priori error estimates of the $h$-version are derived. In [12, a one parameter family of discontinuous Galerkin methods which are parametrized by $\theta \in[-1,1]$ is applied to (1.1) and a priori error estimates which are optimal in $h$ and suboptimal in $p$ are derived in broken

Received by the editor April 14, 2006 and, in revised form, February 23, 2007.

2000 Mathematics Subject Classification. Primary 65N12, 65N15, 65N30.

Key words and phrases. $h p$-finite elements, local discontinuous Galerkin method, second order quasilinear elliptic problems, error estimates, order of convergence.

(C)2007 American Mathematical Society 
$H^{1}$ norm. A new mixed local discontinuous Galerkin method is proposed and analyzed in [8] for a class of nonlinear quasi-Newtonian Stokes fluids in which the nonlinearity is assumed to be monotone. In [11, a nonsymmetric interior penalty Galerkin method is applied and analyzed for a class of problems in which nonlinear convection and linear diffusion is considered.

We note that for nonmonotone nonlinear elliptic problems of the following type:

$$
\begin{aligned}
-\nabla \cdot(a(x, u) \nabla u) & =f \text { in } \Omega, \\
u & =g \text { on } \partial \Omega,
\end{aligned}
$$

where $0<\alpha \leq a(x, u) \leq M$, it is difficult to extend the analysis of [7] or [12]. Therefore, an attempt has been made in this paper to study the LDG method for the problem (1.2)-(1.3). We assume that $\Omega$ is a bounded convex polygon in $\mathbb{R}^{2}$ with boundary $\partial \Omega$, and there exist positive constants $\alpha, M$ such that $0<\alpha \leq$ $a(x, u) \leq M, a(\cdot, \cdot)$ is a twice continuously differentiable function in $\bar{\Omega} \times \mathbb{R}$ and all the derivatives of $a(\cdot, \cdot)$ through second order are bounded in $\bar{\Omega} \times \mathbb{R}$. Further, assume that $f \in L^{2}(\Omega), g$ can be extended to $\Omega$ to be in $H^{2}(\Omega)$ and there exists a unique weak solution $u$ of (1.2) -(1.3) such that $u \in H^{2}(\Omega) \cap W^{1, \infty}(\Omega)$. The results of this paper will be valid for nonlinear $a(\cdot, \cdot)$ provided $a(\cdot, \cdot)$ and its derivatives $a_{u}$, $a_{u u}$ are bounded above in a neighbourhood of $u$ (see Remark 3.2). For notational convenience, we write $a(x, u)$ simply as $a(u)$ in the rest of this paper.

In this paper, an $h p$-LDG method is applied to the problem (1.2)-(1.3) and error estimates which are optimal in $h$ and slightly suboptimal in $p$ are derived. The results proved in this paper are the same as in the linear case; see [18. Assuming $h p$-quasiuniformity condition on the mesh, existence of a solution to the discrete problem is proved using the Brouwer fixed point theorem for small $h$ (mesh size). Moreover, the Lipschitz continuity of the discrete solution map shows the uniqueness of the solution of the discrete problem. Note that the present analysis, in general, cannot be applied to nonlinear problems of monotone type [7, [8, [12. Therefore, the extension of the results to more general nonlinear problems which include monotone types as in the above references is the subject of our current research.

The rest of the article is organized as follows. In Section 2, preliminaries and basic results are noted. Section 3 is devoted to the LDG method and a priori error estimates. In Section 4, numerical experiments are conducted to illustrate the theoretical results for two different nonlinear elliptic problems. Finally, in Section 5 , the article is concluded with some possible extensions of our results.

\section{Preliminaries}

Let $T_{h}=\left\{K_{i}: 1 \leq i \leq N_{h}\right\}$ be a shape regular finite element subdivision of $\Omega$, where $K_{i}$ is either a triangle or a rectangle. For a definition of shape regularity, we refer to [10]. Let $h_{i}$ be the diameter of $K_{i}$ and $h=\max \left\{h_{i}: 1 \leq i \leq N_{h}\right\}$. We denote the set of interior edges of $T_{h}$ by $\Gamma_{I}=\left\{e_{i j}: e_{i j}=\partial K_{i} \cap \partial K_{j},\left|e_{i j}\right|>0\right\}$ and boundary edges by $\Gamma_{\partial}=\left\{e_{i \partial}: e_{i \partial}=\partial K_{i} \cap \partial \Omega,\left|e_{i \partial}\right|>0\right\}$, where $\left|e_{k}\right|$ denotes the one dimensional Euclidean measure. Let $\Gamma=\Gamma_{I} \cup \Gamma_{\partial}$. Note that our definition of $e_{k}$ also includes hanging nodes along each side of the finite elements. On this subdivision $T_{h}$, we define the following broken Sobolev spaces:

$$
V=\left\{v \in L^{2}(\Omega):\left.v\right|_{K_{i}} \in H^{1}\left(K_{i}\right), \text { for all } K_{i} \in T_{h}\right\}
$$


and

$$
\mathbf{W}=\left\{\mathbf{w} \in\left(L^{2}(\Omega)\right)^{2}:\left.\mathbf{w}\right|_{K_{i}} \in\left(H^{1}\left(K_{i}\right)\right)^{2}, \text { for all } K_{i} \in T_{h}\right\},
$$

where $H^{1}\left(K_{i}\right)$ is the standard Sobolev space defined on $K_{i}$. The associated broken norm and seminorm are defined, respectively, as

$$
\|v\|_{H^{1}\left(\Omega, T_{h}\right)}=\left(\sum_{i=1}^{N_{h}}\|v\|_{H^{1}\left(K_{i}\right)}^{2}\right)^{1 / 2} \text { and }|v|_{H^{1}\left(\Omega, T_{h}\right)}=\left(\sum_{i=1}^{N_{h}}|v|_{H^{1}\left(K_{i}\right)}^{2}\right)^{1 / 2} .
$$

We denote the $L^{2}$ norm by $\|\cdot\|$.

Let $e_{k} \in \Gamma_{I}$, that is, $e_{k}=\partial K_{i} \cap \partial K_{j}$ for some $i$ and $j$. Let $\nu_{i}$ and $\nu_{j}$ be the outward normals to the boundary $\partial K_{i}$ and $\partial K_{j}$, respectively. On $e_{k}$, we now define the jump and average of $v \in V$ as

$$
[v]=\left.v\right|_{K_{i}} \nu_{i}+\left.v\right|_{K_{j}} \nu_{j},\{v\}=\frac{\left.v\right|_{K_{i}}+\left.v\right|_{K_{j}}}{2},
$$

and the jump and average of $\mathbf{w} \in \mathbf{W}$ as

$$
[\mathbf{w}]=\left.\mathbf{w}\right|_{K_{i}} \cdot \nu_{i}+\left.\mathbf{w}\right|_{K_{j}} \cdot \nu_{j},\{\mathbf{w}\}=\frac{\left.\mathbf{w}\right|_{K_{i}}+\left.\mathbf{w}\right|_{K_{j}}}{2} .
$$

In case $e_{k} \in \Gamma_{\partial}$, that is, there exists $K_{i}$ such that $e_{k}=\partial K_{i} \cap \partial \Omega$, then set for notational convenience, the jump and average of $v \in V$ as

$$
[v]=\left.v\right|_{K_{i} \cap \partial \Omega} \nu,\{v\}=\left.v\right|_{K_{i} \cap \partial \Omega},
$$

and the jump and average of $\mathbf{w} \in \mathbf{W}$ as

$$
[\mathbf{w}]=\left.\mathbf{w}\right|_{K_{i} \cap \partial \Omega} \cdot \nu,\{\mathbf{w}\}=\left.\mathbf{w}\right|_{K_{i} \cap \partial \Omega},
$$

where $\nu$ is the outward normal to the boundary $\partial \Omega$. For $\mathbf{w} \in \mathbf{W}$, we denote $\mathbf{w}^{2}=\mathbf{w} \cdot \mathbf{w}$. Let $\mathrm{P}_{\mathrm{p}_{\mathrm{i}}}\left(\mathrm{K}_{\mathrm{i}}\right)$ be the space of polynomials of total degree less than or equal to $p_{i}$ on each triangle $K_{i} \in T_{h}$ and $Q_{p_{i}}\left(K_{i}\right)$ be the space of polynomials of degree less than or equal to $p_{i}$ in each variable which are defined on the rectangles $K_{i} \in T_{h}$. The discontinuous finite element spaces are considered as

$$
V_{h}=\left\{v_{h} \in L^{2}(\Omega):\left.v_{h}\right|_{K_{i}} \in \mathrm{Z}_{\mathrm{p}_{\mathrm{i}}}\left(\mathrm{K}_{\mathrm{i}}\right)\right\}
$$

and

$$
\mathbf{W}_{h}=\left\{\mathbf{w}_{h} \in\left(L^{2}(\Omega)\right)^{2}:\left.\mathbf{w}_{h}\right|_{K_{i}} \in \mathrm{Z}_{\mathrm{p}_{\mathrm{i}}}\left(\mathrm{K}_{\mathrm{i}}\right)^{2}\right\},
$$

where $p_{i} \geq 1$ and $\mathrm{Z}_{\mathrm{p}_{\mathrm{i}}}\left(\mathrm{K}_{\mathrm{i}}\right)$ is either $\mathrm{P}_{\mathrm{p}_{\mathrm{i}}}\left(\mathrm{K}_{\mathrm{i}}\right)$ or $\mathrm{Q}_{\mathrm{p}_{\mathrm{i}}}\left(\mathrm{K}_{\mathrm{i}}\right)$. For any $e_{k} \in \Gamma_{I}$, there are two elements $K_{i}$ and $K_{j}$ such that $e_{k}=\partial K_{i} \cap \partial K_{j}$. We associate $h_{k}$ and $p_{k}$ to $e_{k}$, where $p_{k}$ is either $p_{i}$ or $p_{j}$ and $h_{k}$ is either $h_{i}$ or $h_{j}$. For $e_{k} \in \Gamma_{\partial}$, since there is one element $K_{i}$ such that $e_{k}=\partial K_{i} \cap \partial \Omega$, we write $p_{k}=p_{i}$ and $h_{k}=h_{i}$.

\section{Assumption (P).}

(1) The finite element subdivision $T_{h}$ satisfies the bounded local variation in the sense that if $\left|\partial K_{i} \cap \partial K_{j}\right|>0$, for any $K_{i}$ and $K_{j} \in T_{h}$, then there exists a constant $\kappa$ independent of $h_{i}$ and $h_{j}$ such that

$$
\frac{h_{i}}{h_{j}} \leq \kappa
$$

In particular, it implies that for any element $K_{i}$ the number of neighboring elements $K_{j} \in T_{h}$ such that $\left|\partial K_{i} \cap \partial K_{j}\right|>0$ is bounded by $N_{\kappa}$ uniformity. 
(2) The discontinuous finite element space $D_{p}\left(T_{h}\right)$ satisfies the following bounded local variation: If $\left|\partial K_{i} \cap \partial K_{j}\right|>0$, for any $K_{i}$ and $K_{j} \in T_{h}$, then there exists a constant $\varrho$ independent of $p_{i}$ and $p_{j}$ such that

$$
\frac{p_{i}}{p_{j}} \leq \varrho,
$$

where |.| denotes the one dimensional Euclidean measure.

Regular subdivision [10 and 1-irregular subdivision [16] are some examples of subdivision $T_{h}$ of $\Omega$ satisfying the assumption $\mathbf{P}(i)$.

Assumption (Q) ( $h p$-quasiuniformity, [16). Along with the assumption (P), we also assume that the subdivision $T_{h}$ and discontinuous space $D_{p}\left(T_{h}\right)$ satisfies the following $h p$ quasi-uniformity:

$$
\left(\max _{1 \leq i \leq N_{h}} \frac{h_{i}}{p_{i}}\right) \leq C_{Q}\left(\min _{1 \leq i \leq N_{h}} \frac{h_{i}}{p_{i}}\right)
$$

where $C_{Q}$ is a positive constant which is independent of $h$ and $p$.

Observe that under the assumption (2.1), the following holds:

$$
\left(\max _{1 \leq i \leq N_{h}} \frac{p_{i}}{h_{i}}\right)\left(\max _{1 \leq i \leq N_{h}} \frac{h_{i}}{p_{i}}\right)=\left(\min _{1 \leq i \leq N_{h}} \frac{h_{i}}{p_{i}}\right)^{-1}\left(\max _{1 \leq i \leq N_{h}} \frac{h_{i}}{p_{i}}\right) \leq C_{Q} .
$$

Finally, for $v \in V$, we define the following mesh dependent norm

$$
\|v\|^{2}=\left(\sum_{i=1}^{N_{h}}\|\nabla v\|_{L^{2}\left(K_{i}\right)}^{2}+\sum_{e_{k} \in \Gamma_{I}} \int_{e_{k}} \frac{p_{k}^{2}}{h_{k}}[v]^{2} d s\right) .
$$

Approximation properties of the finite element spaces. Below, we state without proof a lemma on some approximation properties.

Lemma 2.1. For $\phi \in H^{s}\left(K_{i}\right)^{d}, d=1$, 2, there exists a positive constant $C_{A}$ (depending on $s$ but independent of $\phi, p_{i}$ and $h_{i}$ ) and a sequence $\phi_{p}^{h} \in \mathrm{Z}_{\mathrm{p}_{\mathrm{i}}}\left(K_{i}\right)^{\mathrm{d}}, \mathrm{p}_{\mathrm{i}}=$ $1,2, \ldots$, such that:

(i) for any $0 \leq l \leq s_{i}$,

$$
\left\|\phi-\phi_{p}^{h}\right\|_{H^{l}\left(K_{i}\right)^{d}} \leq C_{A} \frac{h_{i}^{\mu_{i}-l}}{p_{i}^{s_{i}-l}}\|\phi\|_{H^{s_{i}\left(K_{i}\right)^{d}}}
$$

where $\mu_{i}=\min \left(s_{i}, p_{i}+1\right)$;

(ii) for $s_{i}>l+\frac{1}{2}$,

$$
\left\|\phi-\phi_{p}^{h}\right\|_{H^{l}\left(e_{k}\right)^{d}} \leq C_{A} \frac{h_{i}^{\mu_{i}-l-1 / 2}}{p_{i}^{s_{i}-l-1 / 2}}\|\phi\|_{H^{s_{i}\left(K_{i}\right)^{d}}} ;
$$

(iii) for $0 \leq l \leq s_{i}-1+2 / r$,

$$
\left\|\phi-\phi_{p}^{h}\right\|_{W_{r}^{l}\left(K_{i}\right)^{d}} \leq C_{A} \frac{h_{i}^{\mu_{i}-l-1+2 / r}}{p_{i}^{s_{i}-l-1+2 / r}}\|\phi\|_{H^{s_{i}\left(K_{i}\right)^{d}} .} .
$$

The proof of properties (i) and (ii) can be found in [4. Then using properties (1) and (3) in Lemma 1 of [1] and rescaling, see [2], it is easy to derive the property (iii). We now denote $I_{h} \phi=\phi_{p}^{h}$. 
Trace inequality. We shall use the following trace inequality on the finite element spaces. For a proof, we refer to [19].

Lemma 2.2. Let $v_{h} \in \mathrm{Z}_{\mathrm{p}_{\mathrm{i}}}\left(\mathrm{K}_{\mathrm{i}}\right)^{\mathrm{d}}, \mathrm{d}=1$, 2. Then there exists a constant $C_{T}>0$ such that

$$
\left\|\nabla^{l} v_{h}\right\|_{L^{2}\left(e_{k}\right)^{d}} \leq C_{T} p_{i} h_{i}^{-1 / 2}\left\|\nabla^{l} v_{h}\right\|_{L^{2}\left(K_{i}\right)^{d}}, l=0,1 .
$$

Below, we state without proof a lemma on inverse inequality. For a proof, we refer to [15, p.6], [5].

Lemma 2.3 (Inverse inequalities). Let $v_{h} \in \mathrm{Z}_{\mathrm{p}_{\mathrm{i}}}\left(\mathrm{K}_{\mathrm{i}}\right)^{\mathrm{d}}, \mathrm{d}=1$, 2. Then for $r \geq 2$, there exists a constant $C_{I}>0$ such that

$$
\left\|v_{h}\right\|_{L^{r}\left(K_{i}\right)^{d}} \leq C_{I} p_{i}^{1-2 / r} h_{i}^{(2 / r-1)}\left\|v_{h}\right\|_{L^{2}\left(K_{i}\right)^{d}}, l=0,1 .
$$

In this paper, we use the following version of Poincaré type inequalities on $V$. For a proof, we refer to [6], [15].

Lemma 2.4 (Poincaré type inequalities). For $v \in V$, there exists a constant $C_{P}>$ 0 independent of $h$ and $v$ such that for $1 \leq r<\infty$

$$
\|v\|_{L^{r}(\Omega)} \leq C_{P} \mid\|v\| \| \text {. }
$$

Lemma 2.5 ( $L^{2}$-projection $\left.\Pi\right)$. Let $\boldsymbol{\psi} \in H^{s}\left(K_{i}\right)^{2}$ and $\boldsymbol{\psi}_{h}=\Pi \boldsymbol{\psi} \in \mathrm{Z}_{\mathrm{p}_{\mathrm{i}}}\left(\mathrm{K}_{\mathrm{i}}\right)^{2}$ be the $L^{2}$ projection of $\boldsymbol{\psi}$ onto $\mathrm{Z}_{\mathrm{p}_{\mathrm{i}}}\left(\mathrm{K}_{\mathrm{i}}\right)$. Then, the following approximation properties hold:

$$
\left\|\boldsymbol{\psi}-\boldsymbol{\psi}_{h}\right\|_{L^{2}\left(K_{i}\right)^{2}}+\frac{h_{i}^{1 / 2}}{p_{i}}\left\|\boldsymbol{\psi}-\boldsymbol{\psi}_{h}\right\|_{L^{2}\left(\partial K_{i}\right)^{2}} \leq C \frac{h_{i}^{\mu}}{p_{i}^{s}}\|\boldsymbol{\psi}\|_{H^{s}\left(K_{i}\right)^{2}}
$$

and

$$
\left\|\boldsymbol{\psi}-\boldsymbol{\psi}_{h}\right\|_{L^{4}\left(K_{i}\right)^{2}} \leq C \frac{h_{i}^{\mu-1 / 2}}{p_{i}^{s-1 / 2}}\|\boldsymbol{\psi}\|_{H^{s}\left(K_{i}\right)^{2}},
$$

where $\mu=\min \left\{s, p_{i}+1\right\}$.

Proof. First, inequality of the lemma follows from Lemma 2.1 and the trace inequality (2.3). For the estimate of $\left\|\boldsymbol{\psi}-\boldsymbol{\psi}_{h}\right\|_{L^{4}\left(K_{i}\right)^{2}}$, we use inverse inequality (2.4). This completes the proof.

In our subsequent analysis, we use the following Taylor series expansion for $s$ and $\tau \in \mathbb{R}$ :

$$
a(s)=a(\tau)+\tilde{a}_{u}(s)(s-\tau),
$$

where $\tilde{a}_{u}(s)=\int_{0}^{1} a_{u}(\tau+t(s-\tau)) d t$, and

$$
a(s)=a(\tau)+a_{u}(\tau)(s-\tau)+\tilde{a}_{u u}(s)(s-\tau)^{2},
$$

where $\tilde{a}_{u u}(s)=\int_{0}^{1}(1-t) a_{u u}(\tau+t(s-\tau)) d t$. 


\section{Local discontinuous Galerkin (LDG) Method}

The LDG methods were originally initiated for the system of first order hyperbolic problems. To define the method, we rewrite the equation (1.2) as a system of first order equations. We introduce auxiliary variable $\mathbf{q}=\nabla u$ and $\boldsymbol{\sigma}=a(u) \mathbf{q}$ and rewrite (1.2)-(1.3) as:

$$
\begin{aligned}
\mathbf{q} & =\nabla u \text { in } \Omega, \\
\boldsymbol{\sigma} & =a(u) \mathbf{q} \text { in } \Omega, \\
-\nabla \cdot \boldsymbol{\sigma} & =f \text { in } \Omega, \\
u & =g \text { on } \partial \Omega .
\end{aligned}
$$

We multiply the equation (3.1) by $\mathbf{w} \in \mathbf{W}$, the equation (3.2) by $\boldsymbol{\tau} \in \mathbf{W}$ and the equation (3.3) by $v \in V$ and integrate over the element $K \in T_{h}$. Then using the integration by parts formula, we obtain

$$
\begin{gathered}
\int_{K} \mathbf{q} \cdot \mathbf{w} d x+\int_{K} u \nabla \cdot \mathbf{w} d x-\int_{\partial K} u \mathbf{w} \cdot \nu_{K} d s=0, \\
\int_{K} a(u) \mathbf{q} \cdot \boldsymbol{\tau} d x-\int_{K} \boldsymbol{\sigma} \cdot \boldsymbol{\tau} d x=0,
\end{gathered}
$$

and

$$
\int_{K} \boldsymbol{\sigma} \cdot \nabla v d x-\int_{\partial K} \boldsymbol{\sigma} \cdot \nu_{K} v d s=\int_{K} f v d x .
$$

Note that there may be difficulty in defining $u$ and $\mathbf{q}$ on $\partial K$. Therefore, this is just an initial formulation which is helpful in defining the approximate method given below. The approximate solution $\left(u_{h}, \mathbf{q}_{h}, \boldsymbol{\sigma}_{h}\right) \in \mathrm{Z}_{\mathrm{p}}(\mathrm{K}) \times \mathrm{Z}_{\mathrm{p}}(\mathrm{K})^{2} \times \mathrm{Z}_{\mathrm{p}}(\mathrm{K})^{2}$ is defined using above weak formulation, that is, by imposing that for all $K$, for all $\left(v_{h}, \mathbf{w}_{h}, \boldsymbol{\tau}_{h}\right) \in \mathrm{Z}_{\mathrm{p}}(\mathrm{K}) \times \mathrm{Z}_{\mathrm{p}}(\mathrm{K})^{2} \times \mathrm{Z}_{\mathrm{p}}(\mathrm{K})^{2}$,

$$
\begin{gathered}
\int_{K} \mathbf{q}_{h} \cdot \mathbf{w}_{h} d x+\int_{K} u_{h} \nabla \cdot \mathbf{w}_{h} d x-\int_{\partial K} \hat{u} \mathbf{w}_{h} \cdot \nu_{K} d s=0, \\
\int_{K} a\left(u_{h}\right) \mathbf{q}_{h} \cdot \boldsymbol{\tau}_{h}-\int_{K} \boldsymbol{\sigma}_{h} \cdot \boldsymbol{\tau}_{h} d x=0,
\end{gathered}
$$

and

$$
\int_{K} \boldsymbol{\sigma}_{h} \cdot \nabla v_{h} d x-\int_{\partial K} \hat{\boldsymbol{\sigma}} \cdot \nu_{K} v_{h} d s=\int_{K} f v_{h} d x
$$

where the numerical fluxes $\hat{u}$ and $\hat{\boldsymbol{\sigma}}$ have to be suitably chosen in order to ensure the stability of the method and also to improve the order of convergence. As in the case for linear elliptic problems, we use the following choice of numerical fluxes.

If $e_{k} \in \Gamma_{I}$, then the numerical fluxes are defined on $e_{k}$ as:

$$
\begin{aligned}
\hat{u}\left(u_{h}\right) & =\left\{u_{h}\right\}+C_{12} \cdot\left[u_{h}\right], \\
\hat{\boldsymbol{\sigma}}\left(u_{h}, \boldsymbol{\sigma}_{h}\right) & =\left\{\boldsymbol{\sigma}_{h}\right\}-C_{11}\left[u_{h}\right]-C_{12}\left[\boldsymbol{\sigma}_{h}\right],
\end{aligned}
$$

and if $e_{k} \in \Gamma_{\partial}$, then the numerical fluxes are taken as:

$$
\begin{aligned}
\hat{u} & =g, \\
\hat{\boldsymbol{\sigma}} & =\boldsymbol{\sigma}_{h}-C_{11}\left(u_{h}-g\right) \nu,
\end{aligned}
$$


where $\left.C_{11}\right|_{e_{k}}=\beta p_{k}^{2} / h_{k}, \beta>0$, and $C_{12} \in \mathbb{R}^{2}$ on $e_{k} \in \Gamma_{I}$; we set $C_{12}=0$ on $e_{k} \in \Gamma_{\partial}$. The numerical fluxes are conservative since they are single valued on $e_{k} \in \Gamma_{I}$, that is, on $e_{k} \in \Gamma_{I}$,

$$
[\hat{u}]=0,[\hat{\boldsymbol{\sigma}}]=0,
$$

and consistent since the following holds for smooth $u$ and $\mathbf{q}$ :

$$
\begin{aligned}
\hat{u}(u) & =u, \\
\hat{\boldsymbol{\sigma}}(u, \boldsymbol{\sigma}) & =\boldsymbol{\sigma} .
\end{aligned}
$$

We sum (3.8)-3.10) over all elements $K \in T_{h}$. Then using the conservative property (3.15) and the definition of numerical fluxes, we obtain the following equations:

$$
\begin{array}{r}
\int_{\Omega} \mathbf{q}_{h} \cdot \mathbf{w}_{h} d x+\sum_{i=1}^{N_{h}} \int_{K_{i}} u_{h} \nabla \cdot \mathbf{w}_{h} d x-\int_{\Gamma_{I}}\left(\left\{u_{h}\right\}\right. \\
\left.=C_{12} \cdot\left[u_{h}\right]\right)\left[\mathbf{w}_{h}\right] d s \\
\Gamma_{\partial} g \mathbf{w}_{h} \cdot \nu d s \\
\sum_{i=1}^{N_{h}} \int_{K_{i}} \boldsymbol{\sigma}_{h} \cdot \nabla v_{h} d x-\int_{\Gamma}\left(\left\{\boldsymbol{\sigma}_{h}\right\}-C_{11}\left[u_{h}\right]-C_{12}\left[\boldsymbol{\sigma}_{h}\right]\right)\left[v_{h}\right] d s \\
=\int_{\Omega} f v_{h} d x+\int_{\Gamma_{\partial}} C_{11} g v_{h} d s, \\
\int_{\Omega} a\left(u_{h}\right) \mathbf{q}_{h} \cdot \boldsymbol{\tau}_{h} d x-\int_{\Omega} \boldsymbol{\sigma}_{h} \cdot \boldsymbol{\tau}_{h} d x=0 .
\end{array}
$$

Let $z \in L^{2}(\Omega)$ and $(\phi, \mathbf{p}),(v, \mathbf{w}) \in V \times \mathbf{W}$. We define the following bilinear functional $A_{1}: \mathbf{W} \times \mathbf{W} \rightarrow \mathbb{R}$ as

$$
A_{1}(\mathbf{p}, \mathbf{w})=\int_{\Omega} \mathbf{p} \cdot \mathbf{w} d x
$$

$A_{2}: \mathbf{W} \times V \rightarrow \mathbb{R}$ as

$$
\begin{aligned}
A_{2}(\mathbf{p} ; v) & =\sum_{i=1}^{N_{h}} \int_{K_{i}} \mathbf{p} \cdot \nabla v d x-\int_{\Gamma}\left(\{\mathbf{p}\}-C_{12}[\mathbf{p}]\right)[v] d s \\
& =-\sum_{i=1}^{N_{h}} \int_{K_{i}} v \nabla \cdot \mathbf{p} d x+\int_{\Gamma_{I}}\left(\{v\}+C_{12} \cdot[v]\right)[\mathbf{p}] d s
\end{aligned}
$$

$J: V \times V \rightarrow \mathbb{R}$ as

$$
J(\phi, v)=\int_{\Gamma} C_{11}[\phi][v] d s
$$

and $B: \mathbf{W} \times \mathbf{W} \rightarrow \mathbb{R}$ as

$$
B(z ; \mathbf{p}, \mathbf{w})=\int_{\Omega} a(z) \mathbf{p} \cdot \mathbf{w} d x .
$$

We also define the linear functionals $L_{1}: \mathbf{W} \rightarrow \mathbb{R}$ and $L_{2}: V \rightarrow \mathbb{R}$ as

$$
L_{1}(\mathbf{w})=\int_{\Gamma_{\partial}} g \mathbf{w} \cdot \nu d s \text { and } L_{2}(v)=\int_{\Omega} f v d x+\int_{\Gamma_{\partial}} C_{11} g v d s .
$$


Using the above definitions, we write the LDG method for the problem (3.1)-(3.2) in compact form: Find $\left(u_{h}, \mathbf{q}_{h}, \boldsymbol{\sigma}_{h}\right) \in V_{h} \times \mathbf{W}_{h} \times \mathbf{W}_{h}$ such that for all $\left(v_{h}, \boldsymbol{\tau}_{h}, \mathbf{w}_{h}\right) \in$ $V_{h} \times \mathbf{W}_{h} \times \mathbf{W}_{h}$,

$$
\begin{aligned}
& A_{1}\left(\mathbf{q}_{h}, \mathbf{w}_{h}\right)-A_{2}\left(\mathbf{w}_{h}, u_{h}\right)=L_{1}\left(\mathbf{w}_{h}\right), \\
& A_{2}\left(\boldsymbol{\sigma}_{h}, v_{h}\right)+J\left(u_{h}, v_{h}\right)=L_{2}\left(v_{h}\right), \\
& B\left(u_{h} ; \mathbf{q}_{h}, \boldsymbol{\tau}_{h}\right)-A_{1}\left(\boldsymbol{\sigma}_{h}, \boldsymbol{\tau}_{h}\right)=0 .
\end{aligned}
$$

Since the numerical fluxes $\hat{u}$ and $\hat{\sigma}$ are consistent, we note that the following identity holds for all $(v, \boldsymbol{\tau}, \mathbf{w}) \in V \times \mathbf{W} \times \mathbf{W}$ :

$$
\begin{aligned}
& A_{1}(\mathbf{q}, \mathbf{w})-A_{2}(\mathbf{w}, u)=L_{1}(\mathbf{w}), \\
& A_{2}(\boldsymbol{\sigma}, v)+J(u, v)=L_{2}(v), \\
& B(u ; \mathbf{q}, \boldsymbol{\tau})-A_{1}(\boldsymbol{\sigma}, \boldsymbol{\tau})=0 .
\end{aligned}
$$

In order to derive the a priori error estimates and to prove existence of a unique approximate solution to the problem (3.21)-(3.23), we proceed as follows. Using the equations (3.21)-(3.26) , we write for all $\left(v_{h}, \boldsymbol{\tau}_{h}, \mathbf{w}_{h}\right) \in V_{h} \times \mathbf{W}_{h} \times \mathbf{W}_{h}$ as

$$
\begin{aligned}
& A_{1}\left(\mathbf{q}-\mathbf{q}_{h}, \mathbf{w}_{h}\right)-A_{2}\left(\mathbf{w}_{h}, u-u_{h}\right)=0, \\
& A_{2}\left(\boldsymbol{\sigma}-\boldsymbol{\sigma}_{h}, v_{h}\right)+J\left(u-u_{h}, v_{h}\right)=0, \\
& B\left(u ; \mathbf{q}, \boldsymbol{\tau}_{h}\right)-B\left(u_{h} ; \mathbf{q}_{h}, \boldsymbol{\tau}_{h}\right)-A_{1}\left(\boldsymbol{\sigma}-\boldsymbol{\sigma}_{h}, \boldsymbol{\tau}_{h}\right)=0 .
\end{aligned}
$$

Adding and subtracting $B\left(u ; \mathbf{q}_{h}, \boldsymbol{\tau}_{h}\right)$, we rewrite (3.29) as

$$
B\left(u ; \mathbf{q}-\mathbf{q}_{h}, \boldsymbol{\tau}_{h}\right)-A_{1}\left(\boldsymbol{\sigma}-\boldsymbol{\sigma}_{h}, \boldsymbol{\tau}_{h}\right)=\int_{\Omega}\left(a\left(u_{h}\right)-a(u)\right) \mathbf{q}_{h} \cdot \boldsymbol{\tau}_{h} d x,
$$

and now,

$$
\begin{array}{r}
B\left(u ; \mathbf{q}-\mathbf{q}_{h}, \boldsymbol{\tau}_{h}\right)-A_{1}\left(\boldsymbol{\sigma}-\boldsymbol{\sigma}_{h}, \boldsymbol{\tau}_{h}\right)+\int_{\Omega}\left(a_{u}(u)\left(u-u_{h}\right)\right) \mathbf{q} \cdot \boldsymbol{\tau}_{h} d x \\
=\int_{\Omega}\left(a\left(u_{h}\right)-a(u)\right)\left(\mathbf{q}_{h}-\mathbf{q}\right) \cdot \boldsymbol{\tau}_{h} d x+\int_{\Omega}\left(a\left(u_{h}\right)-a(u)-a_{u}(u)\left(u_{h}-u\right)\right) \mathbf{q} \cdot \boldsymbol{\tau}_{h} d x .
\end{array}
$$

For notational simplicity, we introduce for $\boldsymbol{\tau}, \mathbf{p}, \mathbf{q} \in \mathbf{W}$ and $\phi, v \in V$,

$$
\begin{aligned}
N(u, \mathbf{q} ; \phi, \boldsymbol{\tau}) & =\int_{\Omega}\left(a_{u}(u) \mathbf{q}\right) \phi \cdot \boldsymbol{\tau} d x \\
N_{1}(v-u ; \mathbf{p}-\mathbf{q}, \boldsymbol{\tau}) & =\int_{\Omega}(a(v)-a(u))(\mathbf{p}-\mathbf{q}) \cdot \boldsymbol{\tau} d x \\
& =\int_{\Omega} \tilde{a}_{u}(v)(v-u)(\mathbf{p}-\mathbf{q}) \cdot \boldsymbol{\tau} d x,
\end{aligned}
$$

and

$$
\begin{aligned}
N_{2}(v-u ; \mathbf{q}, \boldsymbol{\tau}) & =\int_{\Omega}\left(a(v)-a(u)-a_{u}(u)(v-u)\right) \mathbf{q} \cdot \boldsymbol{\tau} d x \\
& =\int_{\Omega} \tilde{a}_{u u}(v)(v-u)^{2} \mathbf{q} \cdot \boldsymbol{\tau} d x
\end{aligned}
$$


Hence, the equations (3.27)-3.29) take the form

$$
\begin{aligned}
& A_{1}\left(\mathbf{q}-\mathbf{q}_{h}, \mathbf{w}_{h}\right)-A_{2}\left(\mathbf{w}_{h}, u-u_{h}\right)=0, \quad \mathbf{w}_{h} \in \mathbf{W}_{h}, \\
& A_{2}\left(\boldsymbol{\sigma}-\boldsymbol{\sigma}_{h}, v_{h}\right)+J\left(u-u_{h}, v_{h}\right)=0, \quad v_{h} \in V_{h}, \\
& B\left(u, \mathbf{q}-\mathbf{q}_{h}, \boldsymbol{\tau}_{h}\right)+N\left(u, \mathbf{q} ; u-u_{h}, \boldsymbol{\tau}_{h}\right)-A_{1}\left(\boldsymbol{\sigma}-\boldsymbol{\sigma}_{h}, \boldsymbol{\tau}_{h}\right) \\
& =N_{1}\left(u_{h}-u ; \mathbf{q}_{h}-\mathbf{q}, \boldsymbol{\tau}_{h}\right)+N_{2}\left(u_{h}-u ; \mathbf{q}, \boldsymbol{\tau}_{h}\right), \quad \boldsymbol{\tau}_{h} \in \mathbf{W}_{h} .
\end{aligned}
$$

Now, we prove a coercive type inequality which is useful in our error analysis.

Lemma 3.1. There exist positive constants $C_{1}$ and $C_{2}=C_{2}(u)$ such that for all $(v, \mathbf{w}) \in V \times \mathbf{W}$,

$B(u ; \mathbf{w}, \mathbf{w})+N(u, \mathbf{q} ; v, \mathbf{w})+J(v, v) \geq C_{1}\left(\|\mathbf{w}\|^{2}+\sum_{e_{k} \in \Gamma} \int_{e_{k}} C_{11}[v]^{2} d s\right)-C_{2}\|v\|^{2}$.

Proof. Since $a(u) \geq \alpha>0$, we obtain

$$
B(u ; \mathbf{w}, \mathbf{w})=\int_{\Omega} a(u) \mathbf{w} \cdot \mathbf{w} d x \geq \alpha\|\mathbf{w}\|^{2} .
$$

We note that $\left|a_{u}(u) \mathbf{q}\right|=\left|a_{u}(u) \nabla u\right| \leq M\|u\|_{W^{1, \infty}(\Omega)}$ and

$$
|N(u, \mathbf{q} ; v, \mathbf{w})| \leq M\|u\|_{W^{1, \infty}(\Omega)}\|v\|\|\mathbf{w}\| \leq C(u)\|v\|\|\mathbf{w}\| .
$$

Now, it is easy to see that

$$
\begin{aligned}
B(u ; \mathbf{w}, \mathbf{w})+N(u, \mathbf{q} ; v, \mathbf{w})+J(v, v) & \geq \alpha\|\mathbf{w}\|^{2}+J(v, v)-C(u)\|v\|\|\mathbf{w}\| \\
& \geq \frac{\alpha}{2}\|\mathbf{w}\|^{2}+J(v, v)-\frac{2 C(u)^{2}}{\alpha}\|v\|^{2} .
\end{aligned}
$$

This completes the rest of the proof.

Existence and uniqueness. Below, we recall the Brouwer fixed point theorem [14, p. 218] which is subsequently used to prove the existence of a solution $\left(u_{h}, \mathbf{q}_{h}, \boldsymbol{\sigma}_{h}\right)$ to the discrete problem (3.18)-(3.20).

Theorem 3.2 (Brouwer fixed point theorem). Let $X$ be a finite dimensional Hilbert space and $K$ be a nonempty, convex and compact subset of $X$. Let $\Phi: K \rightarrow K$ be a continuous map. Then, there exists a $v^{*} \in K$ such that $\Phi\left(v^{*}\right)=v^{*}$.

For a given $z \in V_{h}$, we define a map $S_{h}: V_{h} \rightarrow V_{h}$ by $S_{h}(z)=y \in V_{h}$ and $\mathbf{q}_{z}, \boldsymbol{\sigma}_{z} \in \mathbf{W}_{h}$ satisfying

$$
\begin{aligned}
& A_{1}\left(\mathbf{q}-\mathbf{q}_{z}, \mathbf{w}_{h}\right)-A_{2}\left(\mathbf{w}_{h}, u-y\right)=0, \quad \mathbf{w}_{h} \in \mathbf{W}_{h}, \\
& A_{2}\left(\boldsymbol{\sigma}-\boldsymbol{\sigma}_{z}, v_{h}\right)+J\left(u-y, v_{h}\right)=0, \quad v_{h} \in V_{h}, \\
& B\left(u ; \mathbf{q}-\mathbf{q}_{z}, \boldsymbol{\tau}_{h}\right)+N\left(u, \mathbf{q} ; u-y, \boldsymbol{\tau}_{h}\right)-A_{1}\left(\boldsymbol{\sigma}-\boldsymbol{\sigma}_{z}, \boldsymbol{\tau}_{h}\right) \\
& =N_{1}\left(z-u ; \mathbf{q}_{z}-\mathbf{q}, \boldsymbol{\tau}_{h}\right)+N_{2}\left(z-u ; \mathbf{q}, \boldsymbol{\tau}_{h}\right), \boldsymbol{\tau}_{h} \in \mathbf{W}_{h} .
\end{aligned}
$$

Using the definition of $I_{h}$, we write $e_{y}=u-y=\xi_{y}-\eta_{u}$, where $\xi_{y}=I_{h} u-y$ and $\eta_{u}=I_{h} u-u$. Similarly $\mathbf{e}_{q}=\mathbf{q}-\mathbf{q}_{z}=\boldsymbol{\xi}_{q}-\boldsymbol{\eta}_{q}$ and $\mathbf{e}_{\sigma}=\boldsymbol{\sigma}-\boldsymbol{\sigma}_{z}=\boldsymbol{\xi}_{\sigma}-\boldsymbol{\eta}_{\sigma}$, where $\boldsymbol{\xi}_{q}=I_{h} \mathbf{q}-\mathbf{q}_{z}, \boldsymbol{\eta}_{q}=I_{h} \mathbf{q}-\mathbf{q}, \boldsymbol{\xi}_{\sigma}=\Pi \boldsymbol{\sigma}-\boldsymbol{\sigma}_{z}$ and $\boldsymbol{\eta}_{\sigma}=\Pi \boldsymbol{\sigma}-\boldsymbol{\sigma}$. With these 
notations, rewrite (3.33)-(3.35) as

$$
A_{1}\left(\boldsymbol{\xi}_{q}, \mathbf{w}_{h}\right)-A_{2}\left(\mathbf{w}_{h}, \xi_{y}\right)=A_{1}\left(\boldsymbol{\eta}_{q}, \mathbf{w}_{h}\right)-A_{2}\left(\mathbf{w}_{h}, \eta_{u}\right), \quad \forall \mathbf{w}_{h} \in \mathbf{W}_{h},
$$

$$
A_{2}\left(\boldsymbol{\xi}_{\sigma}, v_{h}\right)+J\left(\xi_{y}, v_{h}\right)=A_{2}\left(\boldsymbol{\eta}_{\sigma}, v_{h}\right)+J\left(\eta_{u}, v_{h}\right), \quad v_{h} \in V_{h},
$$

$$
\begin{gathered}
B\left(u ; \boldsymbol{\xi}_{q}, \boldsymbol{\tau}_{h}\right)+N\left(u, \mathbf{q} ; \xi_{y}, \boldsymbol{\tau}_{h}\right)-A_{1}\left(\boldsymbol{\xi}_{\sigma}, \boldsymbol{\tau}_{h}\right)=B\left(u ; \boldsymbol{\eta}_{q}, \boldsymbol{\tau}_{h}\right)+N\left(u, \mathbf{q} ; \eta_{u}, \boldsymbol{\tau}_{h}\right) \\
-A_{1}\left(\boldsymbol{\eta}_{\sigma}, \boldsymbol{\tau}_{h}\right)+N_{1}\left(z-u ; \mathbf{q}_{z}-\mathbf{q}, \boldsymbol{\tau}_{h}\right)+N_{2}\left(z-u ; \mathbf{q}, \boldsymbol{\tau}_{h}\right), \quad \boldsymbol{\tau}_{h} \in \mathbf{W}_{h} .
\end{gathered}
$$

First we show that $S_{h}$ maps from a ball $O_{\delta}\left(I_{h} u\right)$ to itself, where

$$
O_{\delta}\left(I_{h} u\right)=\left\{z \in V_{h}:\left|\left\|z-I_{h} u\right\|\right| \leq \delta\right\}
$$

and for $\epsilon>0$,

$$
\delta=\frac{1}{h^{\epsilon}}\left(\left\|\left|\eta_{u}\left\|\left.\right|^{2}+\right\| \boldsymbol{\eta}_{q}\left\|^{2}+\right\| \boldsymbol{\eta}_{\sigma} \|^{2}+\sum_{e_{k} \in \Gamma} \int_{e_{k}} \frac{h_{k}}{p_{k}^{2}}\left\{\left|\boldsymbol{\eta}_{\sigma}\right|\right\}^{2} d s\right)^{1 / 2} .\right.\right.
$$

With a series of lemmas and theorems, we prove existence and uniqueness results. In Lemma 3.3. we estimate the interpolation errors $\eta_{u}, \boldsymbol{\eta}_{q}$, and $\boldsymbol{\eta}_{\sigma}$. The nonlinear terms $N_{1}$ and $N_{2}$ are estimated in Lemma 3.4 and Lemma 3.5, and are used in proving Theorem 3.7. Theorem 3.8 and Theorem 3.9. To avoid repetition of calculations, we prove Lemma 3.6 which is used subsequently in the proofs of Theorem 3.7 and Theorem 3.9. In Theorem 3.7 and Theorem 3.8 we estimate the errors $\left\|\boldsymbol{\xi}_{\sigma}\right\|$ and $\left\|e_{y}\right\|$, respectively. We then verify the conditions of the Brouwer fixed point theorem for $S_{h}$ in Theorem 3.9 and Theorem 3.10 .

In the following lemma, we estimate the interpolation errors. The proof is an easy consequence of Lemma 2.1 and Lemma 2.5 and is, hence, omitted.

Lemma 3.3. There is a constant $C$ which is independent of $h$ and $p$ such that

$$
\begin{aligned}
\left(\|\| \eta_{u}\|\|^{2}+\left\|\boldsymbol{\eta}_{q}\right\|^{2}+\left\|\boldsymbol{\eta}_{\sigma}\right\|^{2}+\sum_{e_{k} \in \Gamma} \int_{e_{k}} \frac{h_{k}}{p_{k}^{2}}\left\{\left|\boldsymbol{\eta}_{\sigma}\right|\right\}^{2} d s\right) & \leq C\left(\sum_{i=1}^{N_{h}} \frac{h_{i}^{2 \mu_{i}^{+}}}{p_{i}^{2 s_{i}}}\|\nabla u\|_{s_{i}}^{2}\right) \\
& +C\left(\sum_{i=1}^{N_{h}} \frac{h_{i}^{2 \mu_{i}^{*}}}{p_{i}^{2 s_{i}-1}}\|u\|_{s_{i}+1}^{2}\right),
\end{aligned}
$$

where $\mu_{i}^{+}=\min \left\{s_{i}, p_{i}+1\right\}$ and $\mu_{i}^{*}=\min \left\{s_{i}, p_{i}\right\}$.

Since $u \in H^{2}(\Omega)$, using Lemma 3.3 , it is easy to see that

$$
\delta \leq C\left(\|u\|_{H^{2}(\Omega)}\right) \frac{1}{h^{\epsilon}}\left(\max _{1 \leq i \leq N_{h}} h_{i} / p_{i}^{1 / 2}\right) .
$$

In the following lemma, we derive bounds for the nonlinear terms $N_{1}$ and $N_{2}$.

Lemma 3.4. Let Assumption (Q) hold and $z \in O_{\delta}\left(I_{h} u\right)$. For any $0<\epsilon<1 / 2$, there exists a constant $C$ such that

$$
\left|N_{1}\left(z-u ; \mathbf{e}_{q}, \boldsymbol{\tau}\right)+N_{2}(z-u ; \mathbf{q}, \tau)\right| \leq C\left(h^{1 / 2-\epsilon}\left\|\boldsymbol{\xi}_{q}\right\|+h^{1 / 2-\epsilon} \delta\right)\|\boldsymbol{\tau}\| .
$$


Proof. First, we consider the first term on the left hand side of (3.41) and rewrite it as

$$
\begin{aligned}
N_{1}\left(z-u ; \mathbf{q}_{z}-\mathbf{q}, \boldsymbol{\tau}\right)= & \int_{\Omega} \tilde{a}_{u}(z)(z-u)\left(\mathbf{q}_{z}-\mathbf{q}\right) \cdot \boldsymbol{\tau} d x \\
= & -\int_{\Omega} \tilde{a}_{u}(z)\left(z-I_{h} u\right) \boldsymbol{\xi}_{q} \cdot \boldsymbol{\tau} d x+\int_{\Omega} \tilde{a}_{u}(z)\left(z-I_{h} u\right) \boldsymbol{\eta}_{q} \cdot \boldsymbol{\tau} d x \\
& -\int_{\Omega} \tilde{a}_{u}(z) \eta_{u} \boldsymbol{\xi}_{q} \cdot \boldsymbol{\tau} d x+\int_{\Omega} \tilde{a}_{u}(z) \eta_{u} \boldsymbol{\eta}_{q} \cdot \boldsymbol{\tau} d x .
\end{aligned}
$$

Using inverse inequality (2.4) and Lemma 2.1, we estimate the first term on the right hand side of (3.42) as

$$
\begin{aligned}
\left|\int_{\Omega} \tilde{a}_{u}(z)\left(z-I_{h} u\right) \boldsymbol{\xi}_{q} \cdot \boldsymbol{\tau} d x\right| & \leq C \sum_{i=1}^{N_{h}}\left\|z-I_{h} u\right\|_{L^{4}\left(K_{i}\right)}\left\|\boldsymbol{\xi}_{q}\right\|_{L^{4}\left(K_{i}\right)^{2}}\|\boldsymbol{\tau}\|_{L^{2}\left(K_{i}\right)^{2}} \\
& \leq C \sum_{i=1}^{N_{h}} \frac{p_{i}^{1 / 2}}{h_{i}^{1 / 2}}\left\|z-I_{h} u\right\|_{L^{4}\left(K_{i}\right)}\left\|\boldsymbol{\xi}_{q}\right\|_{L^{2}\left(K_{i}\right)^{2}}\|\boldsymbol{\tau}\|_{L^{2}\left(K_{i}\right)^{2}} \\
& \leq C\left(\max _{1 \leq i \leq N_{h}} \frac{p_{i}^{1 / 2}}{h_{i}^{1 / 2}}\right)\left\|z-I_{h} u\right\|\|\| \boldsymbol{\xi}_{q}\|\| \boldsymbol{\tau} \| \\
& \leq C\left(\max _{1 \leq i \leq N_{h}} \frac{p_{i}^{1 / 2}}{h_{i}^{1 / 2}}\right) \frac{1}{h^{\epsilon}}\left(\max _{1 \leq i \leq N_{h}} \frac{h_{i}}{p_{i}^{1 / 2}}\right)\left\|\boldsymbol{\xi}_{q}\right\|\|\boldsymbol{\tau}\| \\
& \leq C h^{1 / 2-\epsilon}\left\|\boldsymbol{\xi}_{q}\right\|\|\boldsymbol{\tau}\| .
\end{aligned}
$$

For the second term on the right hand side of (3.42), use Lemma (2.5) and trace inequality (2.3) to obtain

$$
\begin{aligned}
\left|\int_{\Omega} \tilde{a}_{u}(z)\left(z-I_{h} u\right) \boldsymbol{\eta}_{q} \cdot \boldsymbol{\tau} d x\right| & \leq C \sum_{i=1}^{N_{h}}\left\|z-I_{h} u\right\|_{L^{4}\left(K_{i}\right)}\left\|\boldsymbol{\eta}_{q}\right\|_{L^{4}\left(K_{i}\right)^{2}}\|\boldsymbol{\tau}\|_{L^{2}\left(K_{i}\right)^{2}} \\
& \leq C \sum_{i=1}^{N_{h}} \frac{h_{i}^{1 / 2}}{p_{i}^{1 / 2}}\left\|z-I_{h} u\right\|_{L^{4}\left(K_{i}\right)}\|\mathbf{q}\|_{H^{1}\left(K_{i}\right)^{2}}\|\boldsymbol{\tau}\|_{L^{2}\left(K_{i}\right)^{2}} \\
& \leq C\left(\max _{1 \leq i \leq N_{h}} \frac{h_{i}^{1 / 2}}{p_{i}^{1 / 2}}\right)\left\|z-I_{h} u\right\|\|\mathbf{q}\|_{H^{1}(\Omega)^{2}}\|\boldsymbol{\tau}\| \\
& \leq C h^{1 / 2} \delta\|\boldsymbol{\tau}\| .
\end{aligned}
$$

Similarly, using inverse inequality (2.4) and Lemma 2.1, the third term on the right hand side of (3.42) is estimated as

$$
\begin{aligned}
\left|\int_{\Omega} \tilde{a}_{u}(z) \eta_{u} \boldsymbol{\xi}_{q} \cdot \boldsymbol{\tau} d x\right| & \leq \sum_{i=1}^{N_{h}}\left\|\eta_{u}\right\|_{L^{4}\left(K_{i}\right)}\left\|\boldsymbol{\xi}_{q}\right\|_{L^{4}\left(K_{i}\right)^{2}}\|\boldsymbol{\tau}\|_{L^{2}\left(K_{i}\right)^{2}} \\
& \leq C \sum_{i=1}^{N_{h}} \frac{h_{i}^{3 / 2}}{p_{i}^{3 / 2}}\|u\|_{H^{2}\left(K_{i}\right)} \frac{p_{i}^{1 / 2}}{h_{i}^{1 / 2}}\left\|\boldsymbol{\xi}_{q}\right\|_{L^{2}\left(K_{i}\right)^{2}}\|\boldsymbol{\tau}\|_{L^{2}\left(K_{i}\right)^{2}} \\
& \leq C h\left\|\boldsymbol{\xi}_{q}\right\|\|\boldsymbol{\tau}\| .
\end{aligned}
$$


Then, using Lemma 2.1 we bound the fourth term on the right hand side of (3.42) as

$$
\begin{aligned}
\left|\int_{\Omega} \tilde{a}_{u}(z) \eta_{u} \boldsymbol{\eta}_{q} \cdot \boldsymbol{\tau} d x\right| & \leq \sum_{i=1}^{N_{h}}\left\|\eta_{u}\right\|_{L^{4}\left(K_{i}\right)}\left\|\boldsymbol{\eta}_{q}\right\|_{L^{4}\left(K_{i}\right)^{2}}\|\boldsymbol{\tau}\|_{L^{2}\left(K_{i}\right)^{2}} \\
& \leq C \sum_{i=1}^{N_{h}} \frac{h_{i}^{1-1 / 2}}{p_{i}^{1-1 / 2}}\left\|\eta_{u}\right\|_{L^{4}\left(K_{i}\right)}\|\mathbf{q}\|_{H^{1}\left(K_{i}\right)}\|\boldsymbol{\tau}\|_{L^{2}\left(K_{i}\right)^{2}} \\
& \leq C h^{1 / 2}\left\|\eta_{u}\right\|\|\boldsymbol{\tau}\| \\
& \leq C h^{1 / 2+\epsilon} \delta\|\boldsymbol{\tau}\| .
\end{aligned}
$$

Finally, consider the second term on the left hand side of (3.41). A use of Hölder's inequality with Poincaré type inequality yields

$$
\begin{aligned}
\left|\int_{\Omega} \tilde{a}_{u u}(z) \eta_{u}^{2} \mathbf{q} \cdot \boldsymbol{\tau} d x\right| & \leq C \sum_{i=1}^{N_{h}}\left\|\eta_{u}\right\|_{L^{4}\left(K_{i}\right)}^{2}\|\boldsymbol{\tau}\|_{L^{2}\left(K_{i}\right)^{2}} \\
& \leq C\|\| \eta_{u}\left\|\left.\right|^{2}\right\| \boldsymbol{\tau} \|_{L^{2}\left(K_{i}\right)^{2}} \\
& \leq C h\left\|\eta_{u}\right\|\|\boldsymbol{\tau}\| \\
& \leq C h \delta\|\boldsymbol{\tau}\| .
\end{aligned}
$$

Now combine (3.42)-(3.47) to complete the rest of the proof for any $0<\epsilon<1 / 2$.

In the following lemma, an estimate for the nonlinear terms $N_{1}$ and $N_{2}$ is derived. This is used in the proofs of Theorem 3.8 and Theorem 3.10,

Lemma 3.5. Let $z \in O_{\delta}\left(I_{h} u\right)$. Then, there exists a constant $C$ such that

$$
\begin{aligned}
\left|N_{1}\left(z-u ; \mathbf{e}_{q}, \boldsymbol{\tau}\right)+N_{2}(z-u ; \mathbf{q}, \boldsymbol{\tau})\right| \leq & C|\|z-u\||^{2}\|\boldsymbol{\tau}\|_{L^{4}(\Omega)} \\
& +C\left\|\mathbf{e}_{q}\right\|\|z-u\|\|\boldsymbol{\tau}\|_{L^{4}(\Omega)} .
\end{aligned}
$$

Proof. Consider the first term on the left hand side of (3.48). Using the arguments as in Lemma 3.4 we arrive at

$$
\begin{aligned}
\left|N_{1}\left(z-u ; \mathbf{q}_{z}-\mathbf{q}, \boldsymbol{\tau}\right)\right| & =\left|\int_{\Omega} \tilde{a}_{u}(z)(z-u)\left(\mathbf{q}_{z}-\mathbf{q}\right) \cdot \boldsymbol{\tau} d x\right| \\
& \leq C \mid\|z-u\|\|\| \mathbf{e}_{q}\|\| \boldsymbol{\tau} \|_{L^{4}(\Omega)^{2}} .
\end{aligned}
$$

Next, consider the second term on the left hand side of (3.48). Using Hölder's inequality and Poincaré type inequality we bound the term as:

$$
\begin{aligned}
\left|N_{2}(z-u ; \mathbf{q}, \boldsymbol{\tau})\right| & =\left|\int_{\Omega} \tilde{a}_{u u}(z)(z-u)^{2} \mathbf{q} \cdot \boldsymbol{\tau} d x\right| \\
& \leq C\|z-u\|_{L^{4}(\Omega)}^{2}\|\mathbf{q}\|_{L^{4}(\Omega)^{2}}\|\boldsymbol{\tau}\|_{L^{4}(\Omega)^{2}} \\
& \leq C \mid\|z-u\|\left\|^{2}\right\| \boldsymbol{\tau} \|_{L^{4}\left(K_{i}\right)^{2}} .
\end{aligned}
$$

We now combine (3.49)-(3.50) to complete the rest of the proof.

Using Lemma 2.1 and Lemma 2.5. we prove the following results which will be useful in proving Theorem 3.7 and Theorem 3.9.

Lemma 3.6. There exists a constant $C$ such that $\left|B\left(u ; \boldsymbol{\eta}_{q}, \boldsymbol{\tau}_{h}\right)+N\left(u, \mathbf{q} ; \eta_{u}, \boldsymbol{\tau}_{h}\right)-A_{1}\left(\boldsymbol{\eta}_{\sigma}, \boldsymbol{\tau}_{h}\right)\right| \leq C\left(\left\|\boldsymbol{\eta}_{q}\right\|+\left\|\eta_{u}\right\|\right)\left\|\boldsymbol{\tau}_{h}\right\|, \boldsymbol{\tau}_{h} \in \mathbf{W}_{h}$, 
and for $\mathbf{w}_{h} \in \mathbf{W}_{h}$

$$
\left|A_{1}\left(\boldsymbol{\eta}_{q}, \mathbf{w}_{h}\right)-A_{2}\left(\mathbf{w}_{h}, \eta_{u}\right)\right| \leq C\left(\left\|\boldsymbol{\eta}_{q}\right\|^{2}+\|\| \eta_{u} \mid \|^{2}\right)^{1 / 2}\left\|\mathbf{w}_{h}\right\| .
$$

Proof. Since $\boldsymbol{\eta}_{\sigma}=\Pi \boldsymbol{\sigma}-\boldsymbol{\sigma}$, where $\Pi \boldsymbol{\sigma}$ is the $L^{2}$ projection of $\boldsymbol{\sigma}$, an appeal to the Cauchy-Schwarz inequality yields the proof of the first inequality of the lemma. For the second inequality, we note from the definition that

$$
\begin{aligned}
A_{1}\left(\boldsymbol{\eta}_{q}, \mathbf{w}_{h}\right)-A_{2}\left(\mathbf{w}_{h}, \eta_{u}\right)= & \int_{\Omega} \boldsymbol{\eta}_{q} \cdot \mathbf{w}_{h} d x+\sum_{i=1}^{N_{h}} \int_{K_{i}} \eta_{u} \nabla \cdot \mathbf{w}_{h} d x-\int_{\Gamma_{I}}\left\{\eta_{u}\right\}\left[\mathbf{w}_{h}\right] d s \\
& -\int_{\Gamma_{I}} C_{12} \cdot\left[\eta_{u}\right]\left[\mathbf{w}_{h}\right] d s .
\end{aligned}
$$

For the second term on the right hand side of (3.51), we integrate by parts to obtain

$$
\sum_{i=1}^{N_{h}} \int_{K_{i}} \eta_{u} \nabla \cdot \mathbf{w}_{h} d x-\int_{\Gamma_{I}}\left\{\eta_{u}\right\}\left[\mathbf{w}_{h}\right] d s=-\sum_{i=1}^{N_{h}} \int_{K_{i}} \nabla \eta_{u} \cdot \mathbf{w}_{h}+\int_{\Gamma}\left[\eta_{u}\right]\left\{\mathbf{w}_{h}\right\} d s,
$$

and hence, using trace inequality (2.3) for $l=0$, we arrive at

$$
\left|\sum_{i=1}^{N_{h}} \int_{K_{i}} \eta_{u} \nabla \cdot \mathbf{w}_{h} d x-\int_{\Gamma_{I}}\left\{\eta_{u}\right\}\left[\mathbf{w}_{h}\right] d s\right| \leq C\left|\left\|\eta_{u} \mid\right\|\left\|\mathbf{w}_{h}\right\| .\right.
$$

A use of the Cauchy-Schwarz inequality implies that

$$
\left|\int_{\Omega} \boldsymbol{\eta}_{q} \cdot \mathbf{w}_{h} d x\right| \leq\left\|\boldsymbol{\eta}_{q}\right\|\left\|\mathbf{w}_{h}\right\|
$$

Next, using trace inequality (2.3), we bound the last term on the right hand side of (3.51) as:

$$
\left|\int_{\Gamma_{I}} C_{12} \cdot\left[\eta_{u}\right]\left[\mathbf{w}_{h}\right] d s\right| \leq C\left(\sum_{e_{k} \in \Gamma_{I}} \int_{e_{k}} \frac{p_{k}^{2}}{h_{k}}\left[\eta_{u}\right]^{2}\right)^{1 / 2}\left\|\boldsymbol{\xi}_{\sigma}\right\| .
$$

We now combine (3.51)-3.54 to complete the proof of the lemma.

Using Lemma 3.4 and Lemma 3.6, we now estimate $\boldsymbol{\xi}_{\sigma}$ in the following theorem.

Theorem 3.7. There is a constant $C$ such that for $0<\epsilon<1 / 2$

$$
\left\|\boldsymbol{\xi}_{\sigma}\right\| \leq C\left(\left\|\boldsymbol{\xi}_{q}\right\|+\left\|\xi_{y}\right\|+\left\|\eta_{u}\right\|+\left\|\boldsymbol{\eta}_{q}\right\|+h^{1 / 2-\epsilon} \delta\right) \text {. }
$$

Proof. Using the equation (3.35), we write

$$
\begin{array}{r}
B\left(u ; \boldsymbol{\xi}_{q}, \boldsymbol{\tau}_{h}\right)+N\left(u, \mathbf{q} ; \xi_{y}, \boldsymbol{\tau}_{h}\right)-A_{1}\left(\boldsymbol{\xi}_{\sigma}, \boldsymbol{\tau}_{h}\right)=B\left(u ; \boldsymbol{\eta}_{q}, \boldsymbol{\tau}_{h}\right)+N\left(u, \mathbf{q} ; \eta_{y}, \boldsymbol{\tau}_{h}\right) \\
3.55) \quad-A_{1}\left(\boldsymbol{\eta}_{\sigma}, \boldsymbol{\tau}_{h}\right)+N_{1}\left(z-u ; \mathbf{e}_{q}, \boldsymbol{\tau}_{h}\right)+N_{2}\left(z-u ; \mathbf{q}, \boldsymbol{\tau}_{h}\right) .
\end{array}
$$

Set $\boldsymbol{\tau}_{h}=\boldsymbol{\xi}_{\sigma}$ in 3.55 to obtain

$$
\begin{array}{ll}
\int_{\Omega} \boldsymbol{\xi}_{\sigma} \cdot \boldsymbol{\xi}_{\sigma} d x= & B\left(u, \boldsymbol{\xi}_{q}, \boldsymbol{\xi}_{q}\right)+N\left(u, \mathbf{q} ; \xi_{y}, \boldsymbol{\xi}_{\sigma}\right)-B\left(u ; \boldsymbol{\eta}_{q}, \boldsymbol{\xi}_{\sigma}\right)-N\left(u, \mathbf{q} ; \eta_{y}, \boldsymbol{\xi}_{\sigma}\right) \\
& +A_{1}\left(\boldsymbol{\eta}_{\sigma}, \boldsymbol{\xi}_{\sigma}\right)-N_{1}\left(z-u ; \mathbf{e}_{q}, \boldsymbol{\xi}_{\sigma}\right)-N_{2}\left(z-u ; \mathbf{q}, \boldsymbol{\xi}_{\sigma}\right) .
\end{array}
$$

Then, a use of Lemma 3.4 and Lemma 3.6 completes the proof of the theorem.

Using Lemma 3.4. Lemma 3.5. Lemma 3.6 and Theorem 3.7 we estimate $\left\|e_{y}\right\|$ in the following theorem. 
Theorem 3.8. Let $z \in O_{\delta}\left(I_{h} u\right)$ and $\left(y, \mathbf{q}_{z}, \boldsymbol{\sigma}_{z}\right) \in V_{h} \times \mathbf{W}_{h} \times \mathbf{W}_{h}$ be the corresponding solution of (3.33)-(3.35). For any $0<\epsilon<1 / 2$ the following estimate holds:

$$
\begin{aligned}
\left\|e_{y}\right\| \leq & C_{1}\left(\max _{1 \leq i \leq N_{h}} \frac{h_{i}}{p_{i}^{1 / 2}}\right)\left(\left\|\boldsymbol{\xi}_{q}\right\|^{2}+\sum_{e_{k} \in \Gamma} \int_{e_{k}} C_{11}\left[\xi_{y}\right]^{2} d s\right)^{1 / 2}+C_{2}\|z-u\|^{2} \\
& +C_{3}\left(h^{\epsilon}+h^{1 / 2-\epsilon}\right)\left(\max _{1 \leq i \leq N_{h}} \frac{h_{i}}{p_{i}^{1 / 2}}\right) \delta+C_{4}\left\|\mathbf{e}_{q}\right\|\|\mid z-u\| \| .
\end{aligned}
$$

Proof. We now apply the duality argument. Consider the following auxiliary problem:

$$
\begin{aligned}
-\nabla \cdot(a(u) \nabla \phi)+a_{u}(u) \nabla u \cdot \nabla \phi & =e_{y} \text { in } \Omega, \\
\phi & =0 \text { on } \partial \Omega
\end{aligned}
$$

which satisfies the elliptic regularity

$$
\|\phi\|_{H^{2}(\Omega)} \leq C\left\|e_{y}\right\| .
$$

In order to write the mixed weak formulation, let $\mathbf{p}=\nabla \phi$ and $-\boldsymbol{\psi}=a(u) \mathbf{p}$. Then, we obtain

$$
\begin{aligned}
\mathbf{p} & =\nabla \phi \text { in } \Omega, \\
-\boldsymbol{\psi} & =a(u) \mathbf{p} \text { in } \Omega, \\
\nabla \cdot \boldsymbol{\psi}+a_{u}(u) \mathbf{q} \cdot \mathbf{p} & =e_{y} \text { in } \Omega .
\end{aligned}
$$

We multiply (3.60) by $e_{y},(3.59)$ by $\mathbf{e}_{q}$ and (3.58) by $\mathbf{e}_{\sigma}$, and then integrate over $\Omega$ to arrive at

$$
\begin{aligned}
\left\|e_{y}\right\|^{2}= & \int_{\Omega} e_{y} \nabla \cdot \boldsymbol{\psi} d x+\int_{\Omega} a_{u}(u) \mathbf{q} e_{y} \cdot \mathbf{p} d x+\int_{\Omega} a(u) \mathbf{p} \cdot \mathbf{e}_{q} d x+\int_{\Omega} \boldsymbol{\psi} \cdot \mathbf{e}_{q} \\
& -\int_{\Omega} \mathbf{p} \cdot \mathbf{e}_{\sigma} d x+\int_{\Omega} \nabla \phi \cdot \mathbf{e}_{\sigma} d x .
\end{aligned}
$$

Since $[\phi]=0,[\boldsymbol{\psi}]=0$ on $e_{k} \in \Gamma_{I}$ and $\phi=0$ on $\partial \Omega$, we write

$$
\begin{aligned}
\left\|e_{y}\right\|^{2}= & A_{1}\left(\mathbf{e}_{q}, \boldsymbol{\psi}\right)-A_{2}\left(\boldsymbol{\psi}, e_{y}\right)+B\left(u ; \mathbf{e}_{q}, \mathbf{p}\right)+N\left(u, \mathbf{q} ; e_{y}, \mathbf{p}\right)-A_{1}\left(\mathbf{e}_{\sigma}, \mathbf{p}\right) \\
& +A_{2}\left(\mathbf{e}_{\sigma}, \phi\right)+J\left(e_{y}, \phi\right) .
\end{aligned}
$$

Then, using equations (3.33)-(3.35), we obtain

$$
\begin{aligned}
\left\|e_{y}\right\|^{2} & =A_{1}\left(\mathbf{e}_{q}, \boldsymbol{\eta}_{\psi}\right)-A_{2}\left(\boldsymbol{\eta}_{\psi}, e_{y}\right)+A_{2}\left(\mathbf{e}_{\sigma}, \eta_{\phi}\right)+B\left(u ; \mathbf{e}_{q}, \boldsymbol{\eta}_{p}\right)-A_{1}\left(\mathbf{e}_{\sigma}, \boldsymbol{\eta}_{p}\right) \\
& +N\left(u, \mathbf{q} ; e_{y}, \boldsymbol{\eta}_{p}\right)+J\left(e_{y}, \eta_{\phi}\right)+N_{1}\left(z-u ; \mathbf{e}_{q}, I_{h} \mathbf{p}\right)+N\left(\mathbf{q} ; z-u, I_{h} \mathbf{p}\right),
\end{aligned}
$$


where $\eta_{\phi}=\phi-I_{h} \phi, \boldsymbol{\eta}_{p}=\mathbf{p}-I_{h} \mathbf{p}$ and $\boldsymbol{\eta}_{\psi}=\boldsymbol{\psi}-\Pi \boldsymbol{\psi}$. We now expand (3.61) to find that

$$
\begin{aligned}
\left\|e_{y}\right\|^{2} & =\int_{\Omega} \mathbf{e}_{q} \cdot \boldsymbol{\eta}_{\psi} d x-\int_{\Omega} \mathbf{e}_{\sigma} \cdot \boldsymbol{\eta}_{p} d x+\sum_{i=1}^{N_{h}} \int_{K_{i}} e_{y} \nabla \cdot \boldsymbol{\eta}_{\psi} d x-\int_{\Gamma_{I}}\left\{e_{y}\right\}\left[\boldsymbol{\eta}_{\psi}\right] d s \\
& +\sum_{i=1}^{N_{h}} \int_{K_{i}} \mathbf{e}_{\sigma} \cdot \nabla \eta_{\phi} d x-\int_{\Gamma}\left(\left\{\mathbf{e}_{\sigma}\right\}-C_{11}\left[e_{y}\right]-C_{12}\left[\mathbf{e}_{\sigma}\right]\right)\left[\eta_{\phi}\right] d s \\
& +\int_{\Omega} a(u) \mathbf{e}_{q} \cdot \boldsymbol{\eta}_{p} d x-\int_{\Gamma_{I}} C_{12} \cdot\left[e_{y}\right]\left[\boldsymbol{\eta}_{\psi}\right] d s+\int_{\Omega} a_{u}(u) \mathbf{q} e_{y} \cdot \boldsymbol{\eta}_{p} d x \\
& -N_{1}\left(z-u ; \mathbf{e}_{q}, I_{h} \mathbf{p}\right)+N_{2}\left(z-u ; \mathbf{q}, I_{h} \mathbf{p}\right) .
\end{aligned}
$$

Since $\Pi \boldsymbol{\psi}$ is the $L^{2}$ projection of $\boldsymbol{\psi}$, we bound the following terms using Lemma 2.5 as:

$$
\begin{aligned}
\mid \sum_{i=1}^{N_{h}} \int_{K_{i}} e_{y} \nabla & \cdot \boldsymbol{\eta}_{\psi} d x-\int_{\Gamma_{I}}\left\{e_{y}\right\}\left[\boldsymbol{\eta}_{\psi}\right] d s|=|-\sum_{i=1}^{N_{h}} \int_{K_{i}} \nabla e_{y} \cdot \boldsymbol{\eta}_{\psi} d x+\int_{\Gamma}\left[e_{y}\right]\left\{\boldsymbol{\eta}_{\psi}\right\} d s \mid \\
= & \left|-\sum_{i=1}^{N_{h}} \int_{K_{i}} \nabla \eta_{u} \cdot \boldsymbol{\eta}_{\psi} d x-\sum_{i=1}^{N_{h}} \int_{K_{i}} \nabla \xi_{y} \cdot \boldsymbol{\eta}_{\psi} d x+\int_{\Gamma}\left[e_{y}\right]\left\{\boldsymbol{\eta}_{\psi}\right\} d s\right| \\
\leq & C\left(\sum_{i=1}^{N_{h}} \frac{h_{i}^{2}}{p_{i}^{2}}\left\|\nabla \eta_{u}\right\|_{L^{2}\left(K_{i}\right)}^{2}\right)^{1 / 2}\|\boldsymbol{\psi}\|_{H^{1}(\Omega)^{2}} \\
& +\sum_{e_{k} \in \Gamma}\left(\int_{e_{k}} \frac{p_{k}^{2}}{h_{k}}\left[e_{y}\right]^{2} d s\right)^{1 / 2}\left(\int_{e_{k}} \frac{h_{k}}{p_{k}^{2}}\left\{\boldsymbol{\eta}_{\psi}\right\}^{2} d s\right)^{1 / 2} \\
\leq & C\left(\sum_{i=1}^{N_{h}} \frac{h_{i}^{2}}{p_{i}^{2}}\left\|\nabla \eta_{u}\right\|_{L^{2}\left(K_{i}\right)}^{2}\right)^{1 / 2}\|\boldsymbol{\psi}\|_{H^{1}(\Omega)^{2}} \\
& +\left(\sum_{e_{k} \in \Gamma} \int_{e_{k}} \frac{h_{k}^{2}}{p_{k}^{2}} \frac{p_{k}^{2}}{h_{k}}\left[e_{u}\right]^{2} d s\right)^{1 / 2}\|\boldsymbol{\psi}\|_{H^{1}(\Omega)^{2} .}
\end{aligned}
$$

Next, using Lemma 2.1, we find that

$$
\left|\int_{\Omega} \mathbf{e}_{q} \cdot \boldsymbol{\eta}_{\psi} d x+\int_{\Omega} a(u) \mathbf{e}_{q} \cdot \boldsymbol{\eta}_{p} d x\right| \leq C\left(\sum_{i=1}^{N_{h}} \frac{h_{i}^{2}}{p_{i}^{2}}\left\|\mathbf{e}_{q}\right\|_{L^{2}\left(K_{i}\right)^{2}}^{2}\right)^{1 / 2}\|\mathbf{p}\|_{H^{1}(\Omega)^{2}} .
$$

Again a use of Lemma 2.1 yields

$$
\left|\sum_{i=1}^{N_{h}} \int_{K_{i}} \mathbf{e}_{\sigma} \cdot \nabla \eta_{\phi} d x-\int_{\Omega} \mathbf{e}_{\sigma} \cdot \boldsymbol{\eta}_{p} d x\right| \leq C\left(\sum_{i=1}^{N_{h}} \frac{h_{i}^{2}}{p_{i}^{2}}\left\|\mathbf{e}_{\sigma}\right\|_{L^{2}\left(K_{i}\right)^{2}}^{2}\right)^{1 / 2}\|\phi\|_{H^{2}(\Omega)}
$$

and

$$
\left|\int_{\Omega} a_{u}(u) \mathbf{q} e_{y} \cdot \boldsymbol{\eta}_{p} d x\right| \leq C\left(\sum_{i=1}^{N_{h}} \frac{h_{i}^{2}}{p_{i}^{2}}\left\|e_{y}\right\|_{L^{2}\left(K_{i}\right)}^{2}\right)^{1 / 2}\|\mathbf{p}\|_{H^{1}(\Omega)^{2}} .
$$


Using Lemma 2.5, we obtain

$$
\begin{aligned}
\left|\int_{\Gamma_{I}} C_{12 \cdot} \cdot\left[e_{y}\right]\left[\boldsymbol{\eta}_{\psi}\right] d s\right| & \leq C \sum_{e_{k} \in \Gamma_{I}}\left(\int_{e_{k}} C_{11}\left[e_{y}\right]^{2} d s\right)^{1 / 2}\left(\int_{e_{k}} \frac{h_{k}}{p_{k}^{2}}\left\{\boldsymbol{\eta}_{\psi}\right\}^{2} d s\right)^{1 / 2} \\
& \leq C\left(\sum_{e_{k} \in \Gamma_{I}} \int_{e_{k}} \frac{h_{k}^{2}}{p_{k}^{2}} C_{11}\left[e_{y}\right]^{2} d s\right)^{1 / 2}\|\boldsymbol{\psi}\|_{H^{1}(\Omega)} .
\end{aligned}
$$

Now an application of Lemma 2.1 with trace inequality (2.3) implies that

$$
\begin{aligned}
\mid \int_{\Gamma}\left(\left\{\mathbf{e}_{\sigma}\right\}\right. & \left.-C_{12}\left[\mathbf{e}_{\sigma}\right]\right)\left[\eta_{\phi}\right] d s \mid \leq C \sum_{e_{k} \in \Gamma}\left(\int_{e_{k}}\left\{\left|\boldsymbol{\xi}_{\sigma}\right|\right\}\left|\left[\eta_{\phi}\right]\right| d s+\int_{e_{k}}\left\{\left|\boldsymbol{\eta}_{\sigma}\right|\right\}\left|\left[\eta_{\phi}\right]\right| d s\right) \\
\leq & C\left(\sum_{e_{k} \in \Gamma} \int_{e_{k}} \frac{h_{k}^{3}}{p_{k}^{3}}\left\{\left|\boldsymbol{\xi}_{\sigma}\right|\right\}^{2} d s+\int_{e_{k}} \frac{h_{k}^{3}}{p_{k}^{3}}\left\{\left|\boldsymbol{\eta}_{\sigma}\right|\right\}^{2} d s\right)^{1 / 2}\|\phi\|_{H^{2}(\Omega)} \\
\leq & C\left(\max _{1 \leq i \leq N_{h}} \frac{h_{i}}{p_{i}^{1 / 2}}\right)\left\|\boldsymbol{\xi}_{\sigma}\right\|\|\phi\|_{H^{2}(\Omega)} \\
& +\left(\sum_{e_{k} \in \Gamma} \int_{e_{k}} \frac{h_{k}^{3}}{p_{k}^{3}}\left\{\left|\boldsymbol{\eta}_{\sigma}\right|\right\}^{2} d s\right)^{1 / 2}\|\phi\|_{H^{2}(\Omega)}
\end{aligned}
$$

and

$$
\begin{aligned}
\left|\int_{\Gamma} C_{11}\left[e_{y}\right]\left[\eta_{\phi}\right] d s\right| & \leq C \sum_{e_{k} \in \Gamma}\left(\int_{e_{k}} C_{11}\left[e_{y}\right]^{2} d s\right)^{1 / 2}\left(\int_{e_{k}} \frac{p_{k}^{2}}{h_{k}}\left[\eta_{\phi}\right]^{2} d s\right)^{1 / 2} \\
& \leq C\left(\sum_{e_{k} \in \Gamma} \int_{e_{k}} \frac{h_{k}^{2}}{p_{k}} C_{11}\left[e_{y}\right]^{2} d s\right)^{1 / 2}\|\phi\|_{H^{2}(\Omega)} .
\end{aligned}
$$

Finally, using Lemma 3.5 and $\left\|I_{h} \mathbf{p}\right\|_{L^{4}(\Omega)^{2}}\|\leq C\| \mathbf{p} \|_{H^{1}(\Omega)^{2}}$, we find that

$$
\begin{aligned}
& \left|N_{1}\left(z-u ; \mathbf{e}_{q}, I_{h} \mathbf{p}\right)+N_{2}\left(z-u ; \mathbf{q}, I_{h} \mathbf{p}\right)\right| \\
& \quad \leq C\left|\|z-u\|\left\|^{2}\right\| \mathbf{p}\left\|_{H^{1}(\Omega)^{2}}+\right\| \mathbf{e}_{q}\|\mid\| z-u\|\|\|\mathbf{p}\|_{H^{1}(\Omega)^{2}} .\right.
\end{aligned}
$$

We combine the estimates (3.63)-(3.70) and then use elliptic regularity (3.57) to obtain

$$
\begin{aligned}
\left\|e_{y}\right\| \leq & C\left(\sum_{i=1}^{N_{h}} \frac{h_{i}^{2}}{p_{i}^{2}}\left\|\boldsymbol{\xi}_{q}\right\|_{L^{2}\left(K_{i}\right)^{2}}^{2}+\sum_{e_{k} \in \Gamma} \int_{e_{k}} \frac{h_{k}^{2}}{p_{k}} C_{11}\left[\xi_{y}\right]^{2} d s\right)^{1 / 2}+C_{2}\|\| z-u \|^{2} \\
& +C_{3}\left(\sum_{i=1}^{N_{h}} \frac{h_{i}^{2}}{p_{i}^{2}}\left(\left\|\nabla \eta_{u}\right\|_{L^{2}\left(K_{i}\right)}^{2}+\left\|\boldsymbol{\eta}_{q}\right\|_{L^{2}\left(K_{i}\right)^{2}}^{2}+\left\|\boldsymbol{\eta}_{\sigma}\right\|_{L^{2}\left(K_{i}\right)^{2}}^{2}\right)\right)^{1 / 2} \\
& +C_{4}\left(\sum_{e_{k} \in \Gamma} \int_{e_{k}} \frac{h_{k}^{2}}{p_{k}} C_{11}\left[\eta_{u}\right]^{2} d s+\int_{e_{k}} \frac{h_{k}^{3}}{p_{k}^{3}}\left\{\left|\boldsymbol{\eta}_{\sigma}\right|\right\}^{2} d s\right)^{1 / 2}+C_{5}\left\|\mathbf{e}_{q}\right\|\|z-u\| \| \\
& +C_{6}\left(\max _{1 \leq i \leq N_{h}} \frac{h_{i}}{p_{i}^{1 / 2}}\right)\left\|\boldsymbol{\xi}_{\sigma}\right\| .
\end{aligned}
$$

Now, a use of Theorem 3.7 completes the proof of the theorem. 
Using Lemma 3.3. Lemma 3.4, Lemma 3.6 and Theorem 3.7 we prove in the following theorem that $S_{h}$ maps $O_{\delta}\left(I_{h} u\right)$ into itself.

Theorem 3.9. For all $0<h<h_{0}$ where $h_{0}<1$, there is a $\delta=\delta(h)>0$ such that $S_{h}$ maps from $O_{\delta}\left(I_{h} u\right)$ into itself.

Proof. Set $v_{h}=\xi_{y}, \boldsymbol{\tau}_{h}=\boldsymbol{\xi}_{q}$ and $\mathbf{w}_{h}=\boldsymbol{\xi}_{\sigma}$ in (3.36)-(3.37). Using Lemma 3.1. we obtain

$$
\begin{array}{r}
C_{1}\left(\left\|\boldsymbol{\xi}_{q}\right\|^{2}+\int_{\Gamma} C_{11}\left[\xi_{y}\right]^{2}\right)-C_{2}\left\|\xi_{y}\right\|^{2} \leq A_{1}\left(\boldsymbol{\xi}_{q}, \boldsymbol{\xi}_{\sigma}\right)-A_{2}\left(\boldsymbol{\xi}_{\sigma}, \xi_{y}\right)+B\left(u ; \boldsymbol{\xi}_{q}, \boldsymbol{\xi}_{q}\right) \\
-A_{2}\left(\boldsymbol{\xi}_{\sigma}, \boldsymbol{\xi}_{q}\right)+N\left(u, \mathbf{q} ; \xi_{y}, \boldsymbol{\xi}_{q}\right)+A_{2}\left(\boldsymbol{\xi}_{\sigma}, \xi_{y}\right)+J\left(\xi_{y}, \xi_{y}\right) \\
=A_{1}\left(\boldsymbol{\eta}_{q}, \boldsymbol{\xi}_{\sigma}\right)-A_{2}\left(\boldsymbol{\xi}_{\sigma}, \eta_{u}\right)+B\left(u ; \boldsymbol{\eta}_{q}, \boldsymbol{\xi}_{q}\right)-A_{1}\left(\boldsymbol{\eta}_{\sigma}, \boldsymbol{\xi}_{q}\right) \\
+N\left(u, \mathbf{q} ; \eta_{u}, \boldsymbol{\xi}_{q}\right)+A_{2}\left(\boldsymbol{\eta}_{\sigma}, \xi_{y}\right)+J\left(\eta_{u}, \xi_{y}\right) \\
+N_{1}\left(z-u ; \mathbf{e}_{q}, \boldsymbol{\xi}_{q}\right)+N_{2}\left(z-u ; \mathbf{q}, \boldsymbol{\xi}_{q}\right) .
\end{array}
$$

From the definition of $A_{2}$ and $J$, we write

$$
\begin{aligned}
A_{2}\left(\boldsymbol{\eta}_{\sigma}, \xi_{y}\right)+J\left(\eta_{u}, \xi_{y}\right)= & \sum_{i=1}^{N_{h}} \int_{K_{i}} \boldsymbol{\eta}_{\sigma} \cdot \nabla \xi_{y} d x \\
& -\int_{\Gamma}\left(\left\{\boldsymbol{\eta}_{\sigma}\right\}-C_{11}\left[\eta_{u}\right]-C_{12}\left[\boldsymbol{\eta}_{\sigma}\right]\right)\left[\xi_{y}\right] d s .
\end{aligned}
$$

Since $\Pi \boldsymbol{\sigma}$ are $L^{2}$ projections of $\boldsymbol{\sigma}$ onto $\mathbf{W}_{h}$, we obtain

$$
\sum_{i=1}^{N_{h}} \int_{K_{i}} \boldsymbol{\eta}_{\sigma} \cdot \nabla \xi_{y} d x=0
$$

Next using trace inequality (2.3) and the assumption that $\left.C_{11}\right|_{e_{k}}=\beta p_{k}^{2} / h_{k}$, we bound the following terms as:

$$
\begin{aligned}
\left|\int_{\Gamma}\left(\left\{\boldsymbol{\eta}_{\sigma}\right\}-C_{11}\left[\eta_{u}\right]-C_{12}\left[\boldsymbol{\eta}_{\sigma}\right]\right)\left[\xi_{y}\right] d s\right| \leq & C\left(\sum_{e_{k} \in \Gamma} \int_{e_{k}} \frac{h_{k}}{p_{k}^{2}}\left\{\left|\boldsymbol{\eta}_{\sigma}\right|\right\}^{2} d s\right)^{1 / 2} J\left(\xi_{y}, \xi_{y}\right) \\
& +C J\left(\eta_{u}, \eta_{u}\right) J\left(\xi_{y}, \xi_{y}\right) .
\end{aligned}
$$

An appeal to Lemma 3.6 with $\boldsymbol{\tau}_{h}=\boldsymbol{\xi}_{q}$ and $\mathbf{w}_{h}=\boldsymbol{\xi}_{\sigma}$ yields

$$
\left|B\left(u ; \boldsymbol{\eta}_{q}, \boldsymbol{\xi}_{q}\right)+N\left(u, \mathbf{q} ; \eta_{u}, \boldsymbol{\xi}_{q}\right)-A_{1}\left(\boldsymbol{\eta}_{\sigma}, \boldsymbol{\xi}_{q}\right)\right| \leq C\left(\left\|\boldsymbol{\eta}_{q}\right\|+\left\|\eta_{u}\right\|\right)\left\|\boldsymbol{\xi}_{q}\right\|
$$

and

$$
\left|A_{1}\left(\boldsymbol{\eta}_{q}, \boldsymbol{\xi}_{\sigma}\right)-A_{2}\left(\boldsymbol{\xi}_{\sigma}, \eta_{u}\right)\right| \leq C\left|\left\|\eta_{u} \mid\right\|\left\|\boldsymbol{\xi}_{\sigma}\right\| .\right.
$$

For the last two terms on the right hand side of (3.72), we set $\boldsymbol{\tau}=\boldsymbol{\xi}_{q}$ in Lemma 3.4 to obtain

$$
\begin{aligned}
\left|N_{1}\left(z-u ; \mathbf{e}_{q}, \boldsymbol{\xi}_{q}\right)+N_{2}\left(z-u ; \mathbf{q}, \boldsymbol{\xi}_{q}\right)\right| \leq & C h^{1 / 2-\epsilon}\left\|\boldsymbol{\xi}_{q}\right\|^{2} \\
& +h^{1 / 2-\epsilon} \delta\left\|\boldsymbol{\xi}_{q}\right\| .
\end{aligned}
$$

From Theorem 3.7, we arrive at

$$
\left\|\boldsymbol{\xi}_{\sigma}\right\| \leq C\left(\left\|\boldsymbol{\xi}_{q}\right\|+\left\|\xi_{y}\right\|+\left\|\eta_{u}\right\|+\left\|\boldsymbol{\eta}_{q}\right\|+\left\|\boldsymbol{\eta}_{\sigma}\right\|+h^{1 / 2-\epsilon} \delta\right)
$$


We now combine the estimates (3.72)-(3.78) and obtain for sufficiently small $h$

$$
\begin{aligned}
\left(\left\|\boldsymbol{\xi}_{q}\right\|^{2}+\int_{\Gamma} C_{11}\left[\xi_{y}\right]^{2}\right) \leq & C_{1}\left(\|\| \eta_{u}\|\|^{2}+\left\|\boldsymbol{\eta}_{q}\right\|^{2}+\left\|\boldsymbol{\eta}_{\sigma}\right\|^{2}+h^{1-2 \epsilon} \delta^{2}\right. \\
& \left.+\sum_{e_{k} \in \Gamma} \int_{e_{k}} \frac{h_{k}}{p_{k}^{2}}\left\{\left|\boldsymbol{\eta}_{\sigma}\right|\right\}^{2} d s\right)+C_{2}\left\|\xi_{y}\right\|^{2} \\
\leq & C_{1}\left(h^{2} \epsilon+h^{1-2 \epsilon}\right) \delta^{2}+C_{2}\left\|\xi_{y}\right\|^{2} .
\end{aligned}
$$

Using Theorem 3.8 and the estimate (3.79), we obtain for sufficiently small $h$,

$$
\left(\left\|\boldsymbol{\xi}_{q}\right\|^{2}+\sum_{e_{k} \in \Gamma} \int_{e_{k}} C_{11}\left[\xi_{y}\right]^{2} d s\right)^{1 / 2} \leq C\left(h^{\epsilon}+h^{1 / 2-\epsilon}+\delta\right) \delta .
$$

Next, set $\mathbf{w}_{h}=\nabla \xi_{y}$ in (3.36) to obtain

$$
\begin{aligned}
\sum_{i=1}^{N_{h}} \int_{K_{i}} \boldsymbol{\xi}_{q} \cdot \nabla \xi_{y} d x+\sum_{i=1}^{N_{h}} \int_{K_{i}} \xi_{y} \nabla \cdot \nabla \xi_{y} d x & -\int_{\Gamma_{I}}\left(\left\{\xi_{y}\right\}+C_{12}\left[\xi_{y}\right]\right)\left[\nabla \xi_{y}\right] d s \\
& =A_{1}\left(\boldsymbol{\eta}_{q}, \nabla \xi_{y}\right)-A_{2}\left(\nabla \xi_{y}, \eta_{u}\right) .
\end{aligned}
$$

An integration by parts yields

$$
\begin{aligned}
\sum_{i=1}^{N_{h}} \int_{K_{i}} \nabla \xi_{y} \cdot \nabla \xi_{y} d x= & -\int_{\Omega} \boldsymbol{\xi}_{q} \cdot \nabla \xi_{y} d x+\int_{\Gamma}\left[\xi_{y}\right]\left\{\nabla \xi_{y}\right\} d s+\int_{\Gamma_{I}} C_{12}\left[\xi_{y}\right]\left[\nabla \xi_{y}\right] d s \\
& -A_{1}\left(\boldsymbol{\eta}_{q}, \nabla \xi_{y}\right)+A_{2}\left(\nabla \xi_{y}, \eta_{u}\right) .
\end{aligned}
$$

Apply trace inequality (2.3) and Lemma 3.6 to obtain

$$
\begin{aligned}
& \left(\sum_{i=1}^{N_{h}} \int_{K_{i}}\left\|\nabla \xi_{y}\right\|_{L^{2}\left(K_{i}\right)}^{2} d x\right)^{1 / 2} \\
& \quad \leq C\left(\left\|\boldsymbol{\xi}_{q}\right\|^{2}+\int_{\Gamma} C_{11}\left[\xi_{y}\right]^{2} d s+\|\| \eta_{u} \|^{2}\right)^{1 / 2} .
\end{aligned}
$$

Hence, using (3.80)-(3.81), we obtain for small $h$ and $0<\delta<1$ with $0<\epsilon<1 / 2$

$$
\left\|\left|\xi_{y}\right|\right\| \leq C\left(h^{\epsilon}+h^{1 / 2-\epsilon}+\delta\right) \delta \leq \delta .
$$

This completes the rest of the proof.

We now prove in the following theorem that $S_{h}$ is Lipschitz continuous.

Theorem 3.10. Let $z_{1}, z_{2} \in O_{\delta}\left(I_{h} u\right)$ with $0<\delta<1$. Then for sufficiently small $h$ and $0<\epsilon<1 / 2$, there exists a constant $C$ such that

$$
\text { || } S_{h} z_{1}-S_{h} z_{2}|| \leq C h^{1 / 2-\epsilon}||\left|z_{1}-z_{2}\right||| .
$$

Proof. Let $y_{i}=S_{h} z_{i}, \mathbf{q}_{z_{i}}=\mathbf{q}_{i}$ and $\boldsymbol{\sigma}_{z_{i}}=\boldsymbol{\sigma}_{i}$, for $i=1$, 2. From Theorem 3.9 and the estimates (3.78) as well as (3.80), it follows that

$$
\left(\left\|y_{i}-I_{h} u\right\|\|+\| \mathbf{q}_{i}-I_{h} \mathbf{q}\|+\| \boldsymbol{\sigma}_{i}-\Pi \boldsymbol{\sigma} \|\right) \leq C\left(h^{\epsilon}+h^{1 / 2-\epsilon}+\delta\right) \delta .
$$


Using (3.33)-(3.35), we note that for any $\left(\mathbf{w}_{h}, v_{h}, \boldsymbol{\tau}_{h}\right) \in \mathbf{W}_{h} \times V_{h} \times \mathbf{W}_{h}$

$$
\begin{array}{r}
A_{1}\left(\mathbf{q}_{1}-\mathbf{q}_{2}, \mathbf{w}_{h}\right)-A_{2}\left(\mathbf{w}_{h}, y_{1}-y_{2}\right)=0, \\
A_{2}\left(\boldsymbol{\sigma}_{1}-\boldsymbol{\sigma}_{2}, v_{h}\right)+J\left(y_{1}-y_{2}, v_{h}\right)=0,
\end{array}
$$

and

$$
\begin{gathered}
B\left(u ; \mathbf{q}_{1}-\mathbf{q}_{2}, \boldsymbol{\tau}_{h}\right)+N\left(u, \mathbf{q} ; y_{1}-y_{2}, \boldsymbol{\tau}_{h}\right)-A_{1}\left(\boldsymbol{\sigma}_{1}-\boldsymbol{\sigma}_{2}, \boldsymbol{\tau}_{h}\right) \\
=\int_{\Omega}\left(a\left(z_{1}\right)-a(u)\right)\left(\mathbf{q}_{1}-\mathbf{q}\right) \cdot \boldsymbol{\tau}_{h} d x+\int_{\Omega}\left(a\left(z_{1}\right)-a(u)-a_{u}(u)\left(z_{1}-u\right)\right) \mathbf{q} \cdot \boldsymbol{\tau}_{h} d x \\
-\int_{\Omega}\left(a\left(z_{2}\right)-a(u)\right)\left(\mathbf{q}_{2}-\mathbf{q}\right) \cdot \boldsymbol{\tau}_{h} d x-\int_{\Omega}\left(a\left(z_{2}\right)-a(u)-a_{u}(u)\left(z_{2}-u\right)\right) \mathbf{q} \cdot \boldsymbol{\tau}_{h} d x .
\end{gathered}
$$

We rewrite (3.84) as

$$
\begin{aligned}
& B\left(u ; \mathbf{q}_{1}-\mathbf{q}_{2}, \boldsymbol{\tau}_{h}\right)+N\left(u, \mathbf{q} ; y_{1}-y_{2}, \boldsymbol{\tau}_{h}\right)-A_{1}\left(\boldsymbol{\sigma}_{1}-\boldsymbol{\sigma}_{2}, \boldsymbol{\tau}_{h}\right) \\
& =\int_{\Omega}\left(a\left(z_{1}\right)-a\left(z_{2}\right)\right)\left(\mathbf{q}_{1}-\mathbf{q}\right) \cdot \boldsymbol{\tau}_{h} d x-\int_{\Omega}\left(a\left(z_{2}\right)-a(u)\right)\left(\mathbf{q}_{1}-\mathbf{q}_{2}\right) \cdot \boldsymbol{\tau}_{h} d x \\
& +\int_{\Omega}\left(a\left(z_{1}\right)-a\left(z_{2}\right)-a_{u}\left(z_{2}\right)\left(z_{1}-z_{2}\right)\right) \mathbf{q} \cot \boldsymbol{\tau}_{h} d x \\
& -\int_{\Omega}\left(a_{u}\left(z_{2}\right)-a_{u}(u)\right)\left(z_{1}-z_{2}\right) \mathbf{q} \cdot \boldsymbol{\tau}_{h} d x .
\end{aligned}
$$

Now using similar arguments as in Theorem 3.9, we first obtain

$$
\begin{aligned}
\left(\left\|\mathbf{q}_{1}-\mathbf{q}_{2}\right\|^{2}+\sum_{e_{k} \in \Gamma} \int_{e_{k}} C_{11}\left[y_{1}-y_{2}\right]^{2} d s\right)^{1 / 2} \leq & C_{1} h^{1 / 2-\epsilon}\left\||| z_{1}-z_{2}\right\| \| \\
& +C_{2}\left\|y_{1}-y_{2}\right\|
\end{aligned}
$$

and

$$
\left\|\boldsymbol{\sigma}_{1}-\boldsymbol{\sigma}_{2}\right\| \leq C_{1} h^{1 / 2-\epsilon}\left\|\left|z_{1}-z_{2}\right|\right\|+C_{2}\left\|y_{1}-y_{2}\right\| .
$$

Then an application of duality argument as in Theorem 3.8 yields

$$
\left\|y_{1}-y_{2}\right\| \leq C h^{1 / 2-\epsilon}\left(\left\|\mathbf{q}_{1}-\mathbf{q}_{2}\right\|^{2}+\sum_{e_{k} \in \Gamma} \int_{e_{k}} C_{11}\left[y_{1}-y_{2}\right]^{2} d s\right)^{1 / 2} .
$$

Since

$$
\left\|\left|y_{1}-y_{2}\right|\right\| \leq C\left(\left\|\mathbf{q}_{1}-\mathbf{q}_{2}\right\|^{2}+\sum_{e_{k} \in \Gamma} \int_{e_{k}} C_{11}\left[y_{1}-y_{2}\right]^{2} d s\right)^{1 / 2},
$$

we combine the estimates (3.85)-(3.88) to complete the rest of the proof.

Now, we can conclude from Theorem 3.10 that the map $S_{h}$ is well defined, i.e., the linearized problem (3.33)-(3.35) is well-posed and continuous in the ball $O_{\delta}\left(I_{h} u\right)$. Hence, an appeal to the Brouwer fixed point theorem, that is, Theorem 3.2 with $X=V_{h}, K=O_{\delta}\left(I_{h} u\right)$ and $\Phi=S_{h}$, implies that $S_{h}$ has a fixed point $u_{h}$ in $O_{\delta}\left(I_{h} u\right)$. Then, using Theorem 3.10, it is easy to see that $u_{h}$ is the unique fixed point in $O_{\delta}\left(I_{h} u\right)$ for small $h$. Moreover, $\left(u_{h}, \mathbf{q}_{h}=\mathbf{q}_{u_{h}}, \boldsymbol{\sigma}_{h}=\boldsymbol{\sigma}_{u_{h}}\right)$ is the unique solution for the problem (3.30)-(3.32). 
A priori error estimates. Note that $u_{h}$ satisfies the estimate (3.82) and Theorem 3.8, and $\mathbf{q}_{h}$ satisfies the estimate (3.80). Hence, by choosing $\epsilon=1 / 4$, we easily prove the following theorem.

Theorem 3.11. There exists a constant $C$ such that for sufficiently small $h$ the following estimates hold:

$$
\begin{aligned}
\left\|u-u_{h}\right\| \|^{2} & \leq C \sum_{i=1}^{N_{h}}\left(\frac{h_{i}^{2 \mu_{i}^{+}}}{p_{i}^{2 s_{i}}}\|\nabla u\|_{H^{s_{i}}\left(K_{i}\right)}^{2}+\frac{h_{i}^{2 \mu_{i}^{*}}}{p_{i}^{2 s_{i}-1}}\|u\|_{H^{s_{i}+1}\left(K_{i}\right)}^{2}\right), \\
\left\|\mathbf{q}-\mathbf{q}_{h}\right\|^{2} & \leq C \sum_{i=1}^{N_{h}}\left(\frac{h_{i}^{2} \mu_{i}^{+}}{p_{i}^{2 s_{i}}}\|\nabla u\|_{H^{s_{i}}\left(K_{i}\right)}^{2}+\frac{h_{i}^{2 \mu_{i}^{*}}}{p_{i}^{2 s_{i}-1}}\|u\|_{H^{s_{i}+1}\left(K_{i}\right)}^{2}\right)
\end{aligned}
$$

and

$$
\left\|u-u_{h}\right\|^{2} \leq C\left(\max _{1 \leq i \leq N_{h}} \frac{h_{i}^{2}}{p_{i}}\right) \sum_{i=1}^{N_{h}}\left(\frac{h_{i}^{2 \mu_{i}^{+}}}{p_{i}^{2 s_{i}}}\|\nabla u\|_{H^{s_{i}}\left(K_{i}\right)}^{2}+\frac{h_{i}^{2 \mu_{i}^{*}}}{p_{i}^{2 s_{i}-1}}\|u\|_{H^{s_{i}+1}\left(K_{i}\right)}^{2}\right),
$$

where $\mu_{i}^{+}=\min \left\{s_{i}, p_{i}+1\right\}$ and $\mu_{i}^{*}=\min \left\{s_{i}, p_{i}\right\}$.

Remark 3.1. Note that the error estimates obtained in the above theorem are optimal in $h$ and suboptimal in $p$. These estimates are exactly same as in the case of linear elliptic problems; see [18.

Remark 3.2. In the proof of Lemma 3.4, Lemma 3.5 and also in the subsequent results in Section 3, we have assumed that the range of $\frac{\partial^{l} a}{\partial u^{l}}(x, v), x \in \bar{\Omega}, v \in \mathbb{R}, l=$ $0,1,2$, is a compact set, say $[m, M] \subset \mathbb{R}$. But, we note that asymptotically only the values of $v \in\left[m_{u}-\delta^{*}, M_{u}+\delta^{*}\right] \subset \mathbb{R}$, where $0<\delta^{*}<1, m_{u}=\inf \{u(x): x \in \bar{\Omega}\}$ and $M_{u}=\sup \{u(x): x \in \bar{\Omega}\}$ are considered to derive the proof of Lemma 3.4. Lemma 3.5 and the subsequent results. To be more precise, the terms $\tilde{a}_{u}(z)$ and $\tilde{a}_{u u}(z), z \in O_{\delta}\left(I_{h} u\right)$ in (3.42) and (3.47) (see the estimates (3.43)-(3.50) $)$ can be estimated as follows. Using inverse inequality [17, p. 916], we obtain

$$
\left\|z-I_{h} u\right\|_{L^{\infty}\left(K_{i}\right)} \leq C p_{i}^{1 / 2} h_{i}^{-1 / 4}\left\|z-I_{h} u\right\|_{L^{8}\left(K_{i}\right)} .
$$

Since $z \in O_{\delta}\left(I_{h} u\right)$ with $\delta$ as in (3.39), we find using (3.89), Lemma 2.4 and Lemma 2.1 that

$$
\begin{aligned}
\|z-u\|_{L^{\infty}(\Omega)} & \leq\left\|z-I_{h} u\right\|_{L^{\infty}(\Omega)}+\left\|I_{h} u-u\right\|_{L^{\infty}(\Omega)} \\
& \leq C\left(\max _{1 \leq i \leq N_{h}} \frac{p_{i}^{1 / 2}}{h_{i}^{1 / 4}}\right)\left\|z-I_{h} u\right\|_{L^{8}(\Omega)}+\left\|I_{h} u-u\right\|_{L^{\infty}(\Omega)} \\
& \leq C\left(\max _{1 \leq i \leq N_{h}} \frac{p_{i}^{1 / 2}}{h_{i}^{1 / 4}}\right)\left\|z-I_{h} u\right\|\|+\| I_{h} u-u \|_{L^{\infty}(\Omega)} \\
& \leq C\left(\|u\|_{H^{2}(\Omega)}\right) h^{-\epsilon}\left(\max _{1 \leq i \leq N_{h}} \frac{p_{i}^{1 / 2}}{h_{i}^{1 / 4}}\right)\left(\max _{1 \leq i \leq N_{h}} \frac{h_{i}}{p_{i}^{1 / 2}}\right)+C \frac{h}{p}\|u\|_{H^{2}(\Omega)} \\
& \leq C h^{3 / 4-\epsilon}\left(\max _{1 \leq i \leq N_{h}} \frac{p_{i}^{1 / 2}}{h_{i}^{1 / 2}}\right)\left(\max _{1 \leq i \leq N_{h}} \frac{h_{i}^{1 / 2}}{p_{i}^{1 / 2}}\right)+C \frac{h}{p}\|u\|_{H^{2}(\Omega)} \\
& \leq C h^{3 / 4-\epsilon}\|u\|_{H^{2}(\Omega)} .
\end{aligned}
$$


Therefore, for sufficiently small $h,\|z\|_{L^{\infty}(\Omega)} \leq \delta^{*}+\|u\|_{L^{\infty}(\Omega)}$, where $0<\delta^{*}<$ 1. Now, since the nonlinear functions $a_{u}$ and $a_{u u}$ are continuous, they map the compact set $\left[m_{u}-\delta^{*}, M_{u}+\delta^{*}\right]$ to a compact set in $\mathbb{R}$ and hence, the results in Lemma 3.4, Lemma 3.5 and the subsequent results in Section 3 remain valid when $a(z), a_{u}(z)$ and $a_{u u}(z)$ are bounded for bounded $u$.

\section{Numerical EXPERIMENTS}

In this section, we discuss some numerical results to illustrate the performance of the LDG method applied to two different types of nonlinear elliptic problems. Since the scheme deals with discontinuous finite element spaces, the global basis functions can have support only on a single finite element. Hence, the assembly of the local matrices to the corresponding global matrices is easier than in the case of the conforming finite element method.

For both the examples, we take $\Omega=(0,1) \times(0,1)$ and $g=0$. The finite element subdivision $T_{h}$ is of uniform triangles and the discontinuous finite element spaces of degree $p=1$ and $p=2\left(p_{i}=p \forall i\right)$. Take the stabilizing parameter $\beta=1$ and set $C_{12}=(1,1)$. The LDG method (3.18)-(3.20) has three unknowns, namely $u_{h}, \mathbf{q}_{h}$ and $\boldsymbol{\sigma}_{h}$. Using (3.18), we first solve $\mathbf{q}_{h}$ in terms of $u_{h}$ to write the system (3.19)-(3.20) in two unknowns $u_{h}$ and $\boldsymbol{\sigma}_{h}$. Then, we apply Newton's method to solve this nonlinear system.

Let $\left\{\boldsymbol{\psi}_{l}\right\}_{l=1}^{N_{w}}$ and $\left\{\phi_{i}\right\}_{i=1}^{N_{v}}$ denote bases for $\mathbf{W}_{h}$ and $V_{h}$ respectively, where $N_{w}$ and $N_{v}$ denote the dimensions of $\mathbf{W}_{h}$ and $V_{h}$. Then, define the following matrices:

$$
A=\left[a_{m l}\right]_{1 \leq m, l \leq N_{w}}, B=\left[b_{l i}\right]_{1 \leq l \leq N_{w}}, 1 \leq i \leq N_{v}, \quad D=\left[d_{i j}\right]_{1 \leq i, j \leq N_{v}}
$$

and the vector

$$
L=\left[l_{i}\right]_{1 \leq i \leq N_{v}, 1}
$$

where

$$
\begin{aligned}
& a_{m l}=\int_{\Omega} \boldsymbol{\psi}_{m} \cdot \boldsymbol{\psi}_{l} d x, b_{l i}=\sum_{i=1}^{N_{h}} \int_{K_{i}} \phi_{i} \nabla \cdot \boldsymbol{\psi}_{l} d x-\sum_{e_{k} \in \Gamma_{I}} \int_{e_{k}}\left(\left\{\phi_{i}\right\}+C_{12}[\phi]\right)\left[\boldsymbol{\psi}_{l}\right] d s \\
& d_{i j}=\sum_{e_{k} \in \Gamma} \int_{e_{k}} C_{11}\left[\phi_{i}\right]\left[\phi_{j}\right] d s, \text { and } l_{i}=\int_{\Omega} f \phi_{i} d x
\end{aligned}
$$

Write

$$
u_{h}=\sum_{i=1}^{N_{v}} \alpha_{i} \phi_{i}, \mathbf{q}_{h}=\sum_{l=1}^{N_{w}} b_{l} \boldsymbol{\psi}_{l} \text { and } \boldsymbol{\sigma}_{h}=\sum_{l=1}^{N_{w}} \gamma_{l} \boldsymbol{\psi}_{l},
$$

where $\boldsymbol{\alpha}=\left[\alpha_{1}, \alpha_{2}, \ldots, \alpha_{N_{v}}\right], \mathbf{b}=\left[b_{1}, b_{2}, \ldots, b_{N_{w}}\right]$ and $\gamma=\left[\gamma_{1}, \gamma_{2}, \ldots, \gamma_{N_{w}}\right]$. Using the bases for $V_{h}$ and $\mathbf{W}_{h}$, (3.18) can be reduced to the following matrix equation:

$$
A \mathbf{b}+B \alpha=0, \text { with } A, B \text { defined in (4.1). }
$$

Since the basis functions $\left\{\boldsymbol{\psi}_{l}\right\}_{l=1}^{N_{w}}$ can be assumed independently in each triangle $K \in T_{h}$, the symmetric positive definite global matrix $[A]$ has the following block diagonal form:

$$
[A]=\left\lceil\left[A_{K_{1}}\right], \ldots,\left[A_{K_{N_{h}}}\right]\right\rfloor,
$$


where only the diagonal entries are shown. The other entries in $[A]$ are null matrices. The element matrices $\left[A_{K_{i}}\right]$ are symmetric and positive definite for $i=1,2, \ldots, N_{h}$, and $[A]^{-1}$ has the block diagonal form

$$
[A]^{-1}=\left[\left[A_{K_{1}}\right]^{-1}, \ldots,\left[A_{K_{N_{h}}}\right]^{-1}\right\rfloor .
$$

From (4.3), it is easy to see that $\mathbf{b}=-A^{-1} B \alpha$. Substituting $\mathbf{b}=-A^{-1} B \alpha$ in (3.19)-(3.20), using (4.1)-(4.2) and the bases for $V_{h}$ and $\mathbf{W}_{h},(3.19)-(3.20)$ can be reformulated as: find $[\gamma, \alpha]^{T}$ such that

$$
\begin{aligned}
& F_{i}^{1}(\gamma, \alpha)=0 \text { for } 1 \leq i \leq N_{v} \\
& F_{l}^{2}(\gamma, \alpha)=0 \text { for } 1 \leq l \leq N_{w}
\end{aligned}
$$

where

$$
\begin{gathered}
F_{i}^{1}(\gamma, \alpha)=\sum_{m=1}^{N_{w}} \gamma_{m}\left(-b_{m i}\right)+\sum_{j=1}^{N_{v}} \alpha_{j} d_{j i}-l_{i}, \\
F_{l}^{2}(\gamma, \alpha)=\int_{\Omega} a\left(\sum_{j=1}^{N_{v}} \alpha_{j} \phi_{j}\right)\left(\sum_{m=1}^{N_{w}}\left[-A^{-1} B \alpha\right]_{m} \boldsymbol{\psi}_{m}\right) \cdot \boldsymbol{\psi}_{l} d x-\sum_{m=1}^{N_{w}} \gamma_{m} a_{m l} \\
\text { and }\left[-A^{-1} B \alpha\right]_{m}=-\sum_{j=1}^{N_{v}}\left(A^{-1} B\right)_{m, j} \alpha_{j} .
\end{gathered}
$$

In order to solve the nonlinear algebraic system, we apply Newton's method. The Jacobian Matrix $J$ of the system takes the form

$$
J=\left[\begin{array}{cc}
-B^{T} & D \\
-A & G
\end{array}\right],
$$

where $G=\left[g_{l i}\right]=\left[\partial F_{l}^{2} / \partial \alpha_{i}\right]$ and $B^{T}$ is the transpose of $B$.

Below, we have discussed two examples; one with $a(u)=1+u^{2}$ and the other with $a(u)=1+u, u \geq 0$. Note that using Remark 3.2, the results of Theorem 3.11 are valid.

Example 1. In this example, we set the nonlinear term $a(u)$ as $1+u^{2}$, and choose the load function $f$ suitably so that the exact solution is $u=x\left(e-e^{x}\right) y\left(e-e^{y}\right)$. The initial guess for Newton's iteration is taken to be the solution of the LDG method corresponding to the linearized problem, i.e., by setting $a(u)=1$. For this example, we consider the approximate solution obtained after 10 iterations. The order of convergence for $e_{u}=u-u_{h}$ and $\mathbf{e}_{q}=\mathbf{q}-\mathbf{q}_{h}$ is computed for the cases $p=1$ and 2. Figures 1 and 2 show the computed order of convergences for $\left\|e_{u}\right\|$ and $\left\|e_{\mathbf{q}}\right\|$, respectively, in the log-log scale. These computed order of convergences match with the theoretical order of convergence derived in the Theorem 3.11

Example 2. Set the nonlinear term $a(u)$ as $1+u$ and choose the load function $f$ so that the exact solution is $u=x^{7 / 2}(1-x) y^{7 / 2}(1-y)$. The initial guess and the number of iterations for Newton's method are taken as in Example 1. We then compute the order of convergence for $e_{u}=u-u_{h}$ and $\mathbf{e}_{q}=\mathbf{q}-\mathbf{q}_{h}$ for the cases $p=1$ and 2. Figures 3 and 4 show the computed order of convergences for $\left\|e_{u}\right\|$ and $\left\|e_{\mathbf{q}}\right\|$, respectively, in the log-log scale. These computed order of convergences match with the theoretical order of convergence obtained in Theorem 3.11 . 


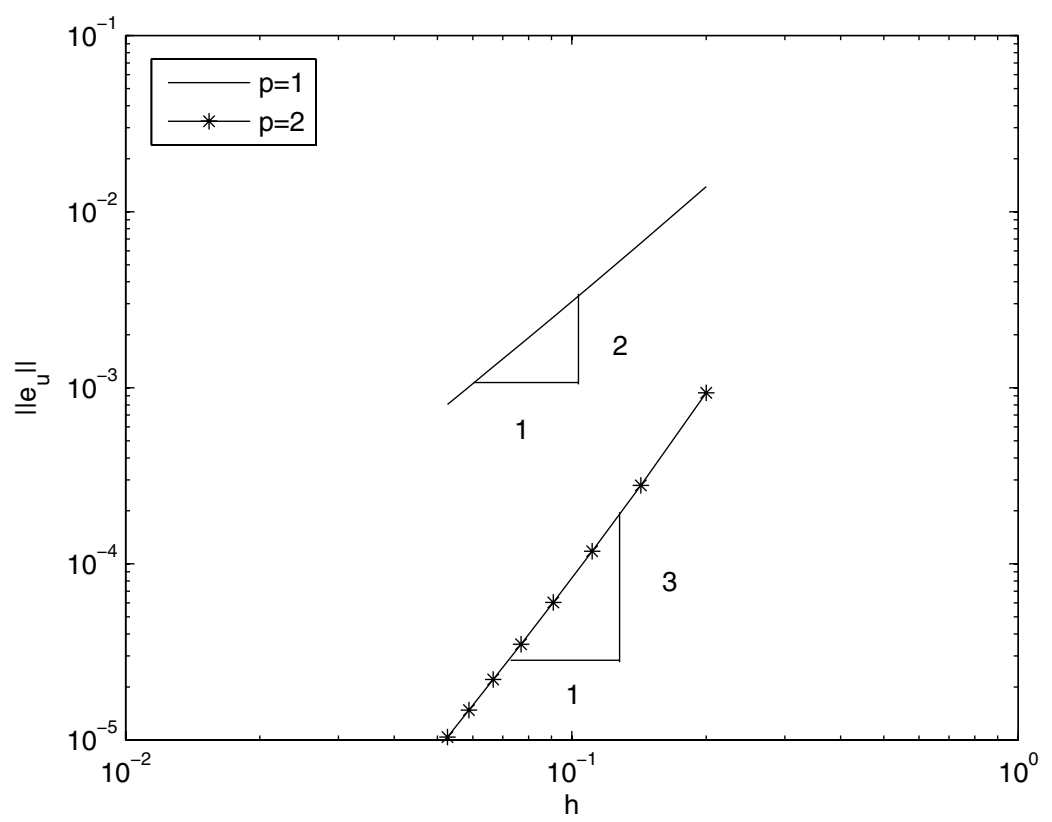

FiguRE 1. Order of convergence for $\left\|e_{u}\right\|$ in Example 1.

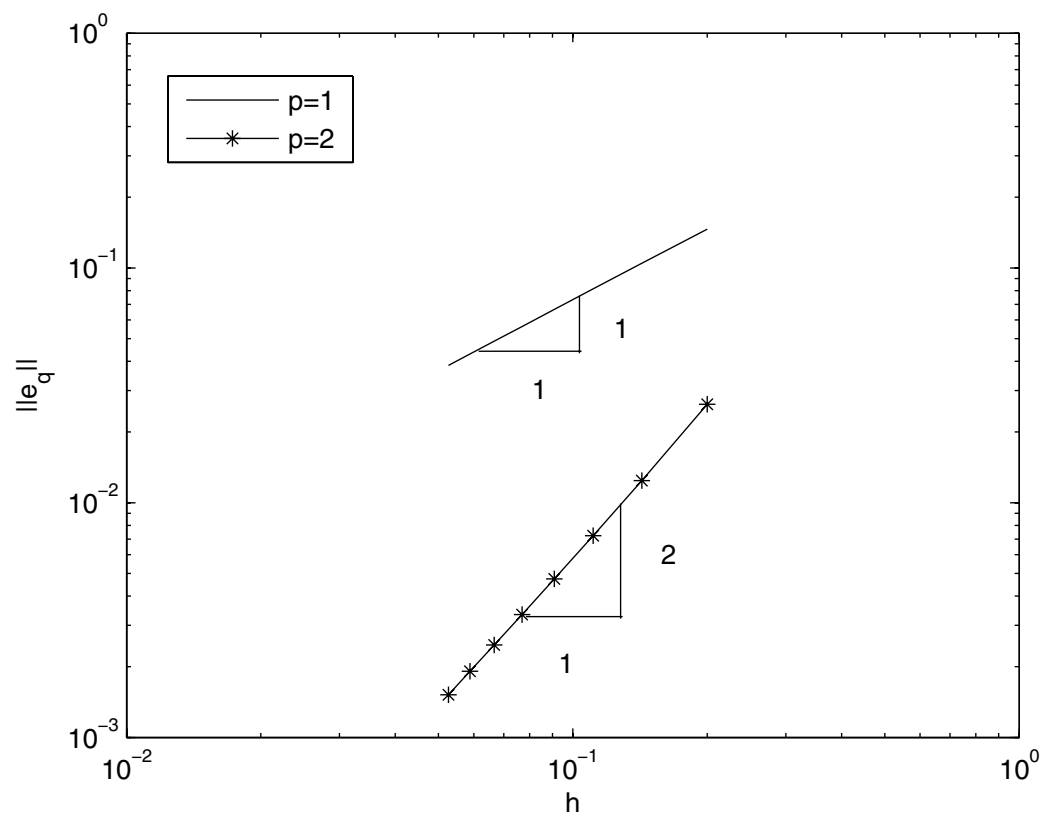

Figure 2. Order of convergence for $\left\|\mathbf{e}_{q}\right\|$ in Example 1. 


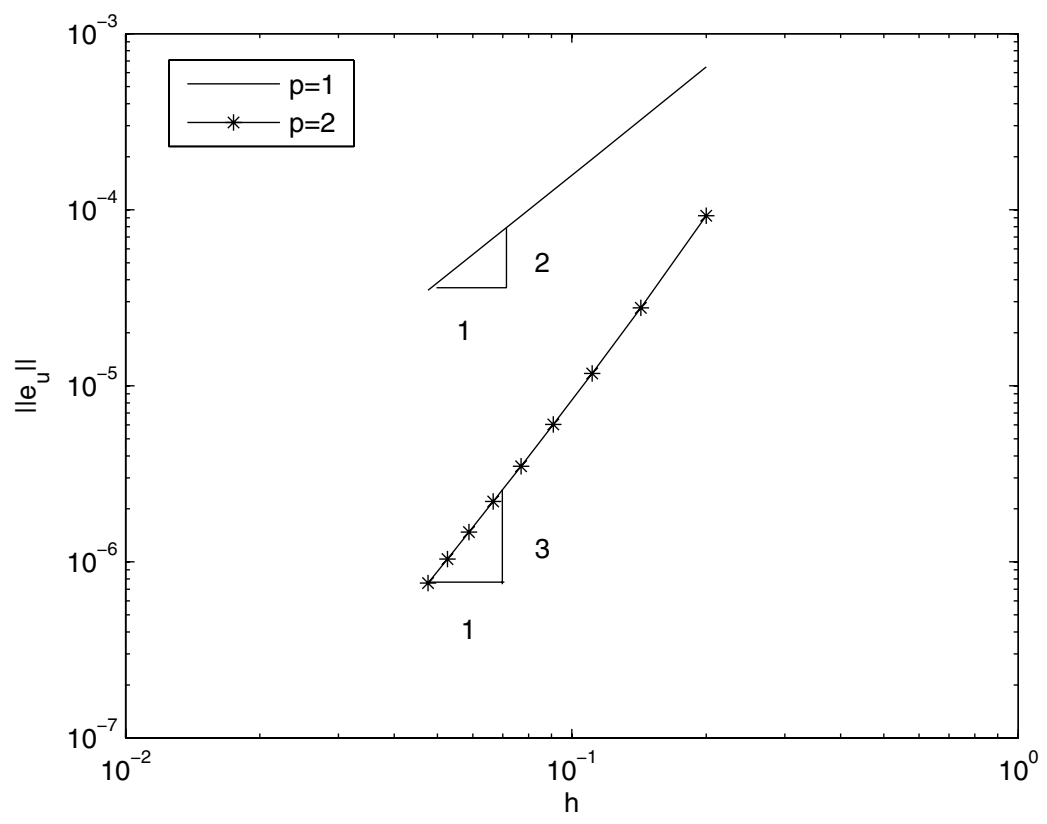

Figure 3. Order of convergence for $\left\|e_{u}\right\|$ in Example 2.

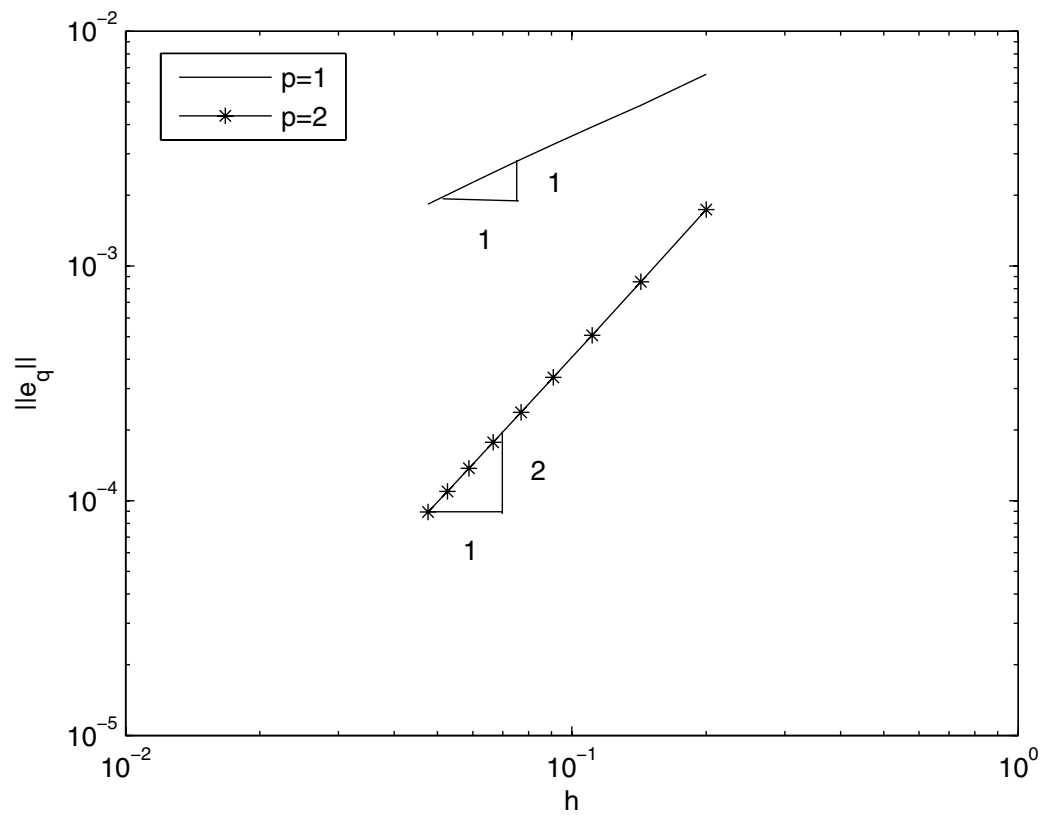

Figure 4. Order of convergence for $\left\|\mathbf{e}_{q}\right\|$ in Example 2. 


\section{Conclusions}

In this paper, we have discussed the $h p$-local discontinuous Galerkin method (LDG) for a class of quasilinear elliptic problems of nonmonotone type. Using the Brouwer fixed point theorem, we have shown that the discrete problem has a solution under $h p$-quasiuniformity assumption on the mesh. Further, using the contraction of the discrete solution map, uniqueness is proved. The error estimate obtained are optimal in $h$ and suboptimal in $p$. These results lead precisely to the same $h$-optimal and mildly $p$-suboptimal rate of convergence as in the case of linear elliptic problems; see [18. The results of this article can easily be extended to the problems in 3 dimension and to the problem $-\nabla \cdot(a(u) \nabla u)+a_{0}(u) u=f(u)$. With appropriate modifications in the analysis, it is possible to extend the theoretical results to the problem (1.2)-(1.3) when $a(u)$ is a bounded uniformly positive definite matrix. The numerical experiments presented in this paper illustrate the performance of the LDG method when it is applied to nonlinear elliptic problems. The extension of the results of the present paper to more general nonlinear boundary value problems is the subject of our current research.

\section{ACKNowledgments}

The authors acknowledge the support of DST and DAAD under the DST-DAAD (PPP-05) project based personal exchange programme.

\section{REFERENCES}

[1] M. Ainsworth and D. Kay, The approximation theory for the p-version finite element method and application to the nonlinear elliptic PDEs, Numer. Math., 82 (1999), 351-388. MR.1692127 (2000i:65177)

[2] M. Ainsworth and D. Kay, Approximation theory for the hp-version finite element method and application to the nonlinear Laplacian, Applied Numerical Mathematics, 34 (2000), 329344. MR1782539(2001e:65175)

[3] D. N. Arnold, F. Brezzi, B. Cockburn and L. D. Marini, Unified analysis of discontinuous Galerkin methods for elliptic problems, SIAM J. Numer. Anal., 39 (2002), 1749-1779. MR.1885715 (2002k:65183)

[4] I. Babuska and M. Suri, The h-p version of the finite element method with quasiuniform meshes, RIARO Model. Math. Anal. Nume., 21 (1987), 199-238. MR896241 (88d:65154)

[5] C. Bernardi, M. Dauge and Y. Maday, Polynomials in the Sobolev world, Preprint of the Laboratoire Jacques-Louis Lions, No. R03038, (2003).

[6] S. C. Brenner, Poincaré-Friedrichs inequalities for piecewise $H^{1}$ functions, SIAM J. Numer. Anal., 41 (2003), 306-324. MR.1974504 (2004d:65140)

[7] R. Bustinza and G. Gatica, A local discontinuous Galerkin method for nonlinear diffusion problems with mixed boundary conditions, SIAM J. Sci. Comput., 26 (2004), 152-177. MR2114338 (2005k:65201)

[8] R. Bustinza and G. Gatica, A mixed local discontinuous Galerkin method for a class of nonlinear problems in fluid mechanics, J. Comput. Phys., 207 (2005), 427-456. MR2144625 (2006a:76069)

[9] P. Castillo, B. Cockburn, I. Perugia and D. Schötzau, An a priori error analysis of the local discontinuous Galerkin method for elliptic problems, SIAM J. Numer. Anal., 38(2000), 16761706. MR1813251 (2002k:65175)

[10] P. G. Ciarlet, The Finite Element Method for Elliptic Problems, North-Holland Publishing Company (1978). MR0520174 (58:25001)

[11] V. Dolejsi, M. Feistauer and V. Sobotikova, Analysis of the discontinuous Galerkin method for nonlinear convection-diffusion problems, Comput. Methods Appl. Mech. Engrg., 194 (2005), 2709-2733. MR2136396 (2005m:65211) 
[12] P. Houston, J. Robson and E. Süli, Discontinuous Galerkin method finite element approximation of quasilinear elliptic boundary value problems I: the scalar case, IMA J. Numer. Anal., 25 (2005), 726-749. MR2170521 (2006k:65322)

[13] J. Douglas and T. Dupont, A Galerkin method for a nonlinear Dirichlet problem, Math. Comp., 29(1975), 689-696. MR0431747 (55:4742)

[14] S. Kesavan, Topics in Functional Analysis and Applications, Wiley-Eastern Ltd., (1989). MR $990018(90 \mathrm{~m}: 46002)$

[15] A. Lasis and E. Süli, Poincaré -Type inequalities for Broken Sobolev spaces, Isaac Newton Institute for Mathematical Sciences, Preprint No. NI03067-CPD, (2003).

[16] A. Lasis and E. Süli, One-parameter discontinuous Galerkin finite element discretisation of quasilinear parabolic problems, Oxford Univ. Comp. Lab., Research Report NA-04/25 (2004).

[17] F. A. Milner and M. Suri, Mixed finite element methods for quasilinear elliptic problems: The p-version, $\mathrm{M}^{2}$ AN, 26 (1992), 913-931. MR1199319 (94f:65104)

[18] I. Perugia and D. Schöetzau, An hp-analysis of the local discontinuous Galerkin method for diffusion problems, J. Sci. Comput., 17 (2001), 561-571. MR.1910752

[19] B. Riviere, M. F. Wheeler, and V. Girault, A priori error estimates for finite element methods based on discontinuous approximation spaces for elliptic problems, SIAM J. Numer. Anal., 39(2001), 902-931. MR1860450 (2002g:65149)

Department of Mathematics, Industrial Mathematics Group, indian Institute of Technology Bombay, Powai, Mumbai-400076

E-mail address: trpathi@math.iitb.ac.in

Department of Mathematics, Industrial Mathematics Group, indian Institute of Technology Bombay, Powai, Mumbai-400076

E-mail address: neela@math.iitb.ac.in

Department of Mathematics, Industrial Mathematics Group, Indian Institute of Technology Bombay, Powai, Mumbai-400076

E-mail address: akp@math.iitb.ac.in 